\title{
Star-unitary transformations. From dynamics to irreversibility and stochastic behavior
}

\author{
Sungyun Kim \\ Center for Studies in Statistical Mechanics and Complex Systems, \\ The University of Texas at Austin, Austin, TX 78712 USA \\ Gonzalo Ordonez \\ Center for Studies in Statistical Mechanics and Complex Systems, \\ The University of Texas at Austin, Austin, TX 78712 USA \\ and International Solvay Institutes for Physics and Chemistry, CP231, 1050 Brussels, Belgium
}

(Dated: October 25, 2018)

\begin{abstract}
We consider a simple model of a classical harmonic oscillator coupled to a field. In standard approaches Langevin-type equations for bare particles are derived from Hamiltonian dynamics. These equations contain memory terms and are time-reversal invariant. In contrast the phenomenological Langevin equations have no memory terms (they are Markovian equations) and give a time evolution split in two branches (semigroups), each of which breaks time symmetry. A standard approach to bridge dynamics with phenomenology is to consider the Markovian approximation of the former. In this paper we present a formulation in terms of dressed particles, which gives exact Markovian equations. We formulate dressed particles for Poincaré nonintegrable systems, through an invertible transformation operator $\Lambda$ introduced by Prigogine and collaborators. $\Lambda$ is obtained by an extension of the canonical (unitary) transformation operator $U$ that eliminates interactions for integrable systems. Our extension is based on the removal of divergences due to Poincaré resonances, which breaks time-symmetry. The unitarity of $U$ is extended to "star-unitarity" for $\Lambda$. We show that $\Lambda$-transformed variables have the same time evolution as stochastic variables obeying Langevin equations, and that $\Lambda$-transformed distribution functions satisfy exact Fokker-Planck equations. The effects of Gaussian white noise are obtained by the non-distributive property of $\Lambda$ with respect to products of dynamical variables. Therefore our method leads to a direct link between dynamics of Poincaré nonintegrable systems, probability and stochasticity.

PACS numbers: 02.50.Fz, 05.40.-a, 05.70.Ln
\end{abstract}

\section{INTRODUCTION}

In classical physics the basic laws are time reversible. If we know the Hamiltonian, then we get Hamilton's equations of motion which describe the time evolution of the system in a time reversible, deterministic way. On the other hand, we see time irreversibility and stochastic behavior everywhere. How to bridge the gap between theory and reality has been the subject of many discussions.

The main problem is how to extract irreversibility and stochasticity out of Hamilton's equations of motion. This will be the subject of this paper. Our approach is an extension of canonical transformations to define dressed particles or quasiparticles [1].

We consider Hamiltonians that can be written as

$$
H=H_{0}+\lambda V \text {. }
$$

The first therm $H_{0}$ describes a set of noninteracting "bare" units while the second $\lambda V$ describes their interactions ( $\lambda$ is a dimensionless coupling constant). Specifically, we will consider the one-dimensional Friedrichs model 2], describing a classical harmonic oscillator (bare particle) coupled to an infinite set of bare field modes (heat bath). This model is closely related to the CaldeiraLeggett model [3], which has been extensively used to study quantum Brownian motion [4, 5, 6, 7, 8, 9].
In general, bare particles follow a complicated motion, due to their interactions. In order gain a physical insight into their behavior, and also to simplify the equations of motion, one can introduce a change of phase-space variables (a canonical transformation). The new variables describe renormalized entities, or quasiparticles. After solving the equations for quasiparticles, one may apply the inverse canonical transformation to get the solutions of the original equations of motion. For the Friedrichs model the quasiparticle consists of the original particle surrounded by a "dressing" cloud of field modes.

For integrable systems, one can construct transformations $U$ that completely eliminate the interactions. They bring us to a description in terms of free quasiparticles. This is readily seen starting with the Liouville equation

$$
i \frac{\partial}{\partial t} \rho=L_{H} \rho
$$

where $L_{H} \equiv i\{H$,$\} is the Poisson bracket with the$ Hamiltonian. Similar to Eq. (1) the Liouvillian is written as a free term plus interaction, $L_{H}=L_{0}+\lambda L_{V}$. Applying $U$ on both sides of the Liouville equation we get

$$
\begin{aligned}
i \frac{\partial}{\partial t} U \rho & =U L_{H} U^{-1} U \rho \\
\Rightarrow i \frac{\partial}{\partial t} \bar{\rho} & =\bar{L}_{0} \bar{\rho}
\end{aligned}
$$


where

$$
\bar{\rho}=U \rho, \quad \overline{L_{0}}=U L_{H} U^{-1} .
$$

The transformation $U$ is constructed in such a way that $\bar{L}_{0}$ has the same form as the non-interacting Liouvillian, with renormalized frequencies. Eq. (3) gives the time evolution of the free dressed particles.

If $U$ can be constructed through a perturbation expansion in $\lambda$, we say the system is integrable in the sense of Poincaré. For these systems we can keep a one-toone correspondence between the original variables and the transformed variables. With a suitably defined inner product between dynamical variables and ensembles, we can define the hermitian conjugate transformation $U^{\dagger}$. One then finds that $U$ is unitary: $U^{\dagger}=U^{-1}$. The transformation $U$ thus preserves the time-reversibility of the original Liouville equation.

Now, if all systems were integrable in Poincaré's sense, this would mean that all the phenomena we observe in nature are equivalent to free motion. This would be hard to reconcile with the existence of dissipative phenomena, which are essential for the appearance of bifurcations and self-organization [10]. However for most systems one cannot construct $U$ by perturbation expansions, due to the appearance of resonances. Resonances give vanishing denominators leading to divergences. These divergences were discovered by Poincaré, so we will refer to them as Poincaré divergences [hereafter, whenever we speak of integrability or nonintegrability, it will be meant in Poincaré's sense].

It is precisely for Poincaré's nonintegrable systems that we see irreversible and stochastic behavior, such as Brownian motion. One of the main developments of the Brussels-Austin groups led by I. Prigogine has been to show that one can systematically eliminate the Poincaré divergences by regularization of denominators 11, 12, 13, 14, 15]. As a result of this regularization time-symmetry is broken and one obtains a new type of transformation $\Lambda$ that replaces $U$. This gives a quasiparticle description leading to stochastic or kinetic equations, such as the classical Langevin or Fokker-Planck equations, respectively. To see this, we operate $\Lambda$ on the Liouville equation

$$
\begin{aligned}
i \frac{\partial}{\partial t} \Lambda \rho & =\Lambda L_{H} \Lambda^{-1} \Lambda \rho \\
\Rightarrow i \frac{\partial}{\partial t} \tilde{\rho} & =\tilde{\theta} \tilde{\rho}
\end{aligned}
$$

where

$$
\tilde{\rho}=\Lambda \rho, \quad \tilde{\theta}=\Lambda L_{H} \Lambda^{-1} .
$$

$\tilde{\theta}$ is now a collision operator as used in kinetic theory. The $\Lambda$ transformation gives a probabilistic description, which is irreducible to trajectories in classical mechanics or wave functions in quantum mechanics. If we integrate out the field variables, Eq. (5) becomes, e.g., an exact Fokker-Planck operator. Through the $\Lambda$ transformation we can also describe dressed unstable states in quantum mechanics [15, 16]. One can define as well an $\mathcal{H}$-function that has strict monotonic behavior [1].

For the Friedrichs model we have both integrable and nonintegrable cases, depending on whether the spectrum of the field modes is discrete (finite volume $L$ with periodic boundaries) or continuous $(L \rightarrow \infty)$. In the first case we have cyclic (although complicated) motion of the particle, as the field comes back to the particle through the periodic boundaries. In the second case, the field does not come back. A Poincaré resonance emerges, since the energy of the particle is embedded inside the continuous spectrum. The emission of the field from the particle leads to radiation damping. Conversely, the particle is excited when it absorbs the field.

To understand the breaking of time symmetry, we note that when there are Poincaré resonances, i.e. in the limit $L \rightarrow \infty$, the solutions of Hamilton's equations for the bare particle contain a dominant decaying (Markovian) component oriented either towards the future or the past, or both, depending on the initial conditions. Taking the well-known Markovian approximation, one finds that the equation of motion for the bare particle is split into two branches, one for $t>0$ and another for $t<0$, corresponding to two semigroups. As a whole the time reversal invariance of the motion is kept, but if we pick either branch, time symmetry is broken.

To obtain this splitting into two semigroups for the bare particle we have to make approximations. In contrast, in terms of the dressed particle defined through $\Lambda$, this is an exact property. The analytic continuation of $U$ can be made to either the upper or lower complex frequency planes, giving exact Markovian equations that generate the $t<0$ or $t>0$ semigroups, respectively. Once we fix the analytic continuation, time symmetry is broken.

The $\Lambda$-transformed functions involve generalized functions, or distributions (examples are the "Gamow modes" presented in Sec. (V). If the initial unperturbed functions formed a Hilbert space, the transformed functions are no more in this Hilbert space. In its transformed domain $L_{H}$ behaves as the dissipative collision operator $\tilde{\theta}$ with complex eigenvalues [13, 14].

In contrast to $U, \Lambda$ is no more unitary. Instead, it is "star unitary" 11, 15]. Furthermore, while $U$ is distributive with respect to multilplication, $\Lambda$ is non-distributive. As we will see, these properties allow us to describe damping and fluctuations associated with noise.

A basic requirement on $\Lambda$ is that it is invertible. This is connected with the star unitarity of this transformation [see comments below Eq. [77)]. In addition to this, our construction of $\Lambda$ is based on the following requirements:

(1) The $\Lambda$ transformation is obtained by analytic continuation of the unitary transformation $U$. When there are no resonances, $\Lambda$ reduces to $U$.

(2) $\Lambda$ preserves the measure of the phase space.

(3) $\Lambda$ maps real variables to real variables.

(4) $\Lambda$ is analytic with respect to the coupling constant $\lambda$ 
at $\lambda=0$.

(5) $\Lambda$ leads to closed Markovian kinetic equations.

We will focus on the dynamical observables of the particle. The action of $\Lambda$ will be restricted to the subset of phase space functions depending only on the particle degrees of freedom. Within this subset we obtain an exact and invertible $\Lambda$ transformation.

Furthermore, we will consider the thermodynamic limit of the field modes, where the total energy of the field is an extensive variable. Then the average action $\left\langle J_{k}\right\rangle$ of each field mode $k$ satisfies [17]

$$
\left\langle J_{k}\right\rangle \sim O\left(L^{0}\right)
$$

for $L \rightarrow \infty$. The total energy of the field

$$
E_{\mathrm{f}}=\sum_{k} \omega_{k}\left\langle J_{k}\right\rangle \rightarrow \frac{L}{2 \pi} \int d k \omega_{k}\left\langle J_{k}\right\rangle \sim O(L)
$$

is proportional to the volume $L$. [This does not necessarily imply that the field is Gibbsian]. The existence of the thermodynamic limit requires an initially random distribution of the phases of the field modes [18].

A different situation occurs if the total energy of the field is in a non-extensive variable. Then we have $\left\langle J_{k}\right\rangle \sim$ $O\left(L^{-1}\right)$, i.e., we have a vanishing energy density. We will not consider this case in this paper.

In the extensive case, in addition to the damped oscillation, the particle undergoes an erratic motion due to the excitation caused by the field. This erratic motion includes a Brownian motion component, which is Markovian. The initial randomness of the phases of the field modes is a necessary condition for the appearance of Brownian motion. In addition it is essential that the field resonates with the particle. We need Poincaré resonances. Under these conditions $\Lambda$ permits us to isolate the damping and the Brownian component of the motion.

Our approach can also be formulated in terms of complete sets of projection operators $\Pi^{(\nu)}$, that permit to decompose dynamics into a set of orthogonal "subdynamics" (see Sec. VI). Essentially, we introduce a generalized basis that permits us to analyze the motion in terms of strictly Markovian components. In our case, we study the component that describes Brownian motion. The other components, clumped together, give what is usually called non-Markovian (memory) effects [14]. The Brownian component is independent of the initial correlations between the particle and the bath, and in this sense, it has a "universal" character.

The results presented here are based on Refs. [15, 16], where we constructed $\Lambda$ for the quantum Friedrichs model. The main subject in these papers was the decay of unstable particle states. We showed that the $\Lambda$ transformation permits us to isolate the exponential (Markovian) component of the decay, which occurs when the energy of the field is non-extensive. The remaining (non-Markovian) component gives the Zeno effect 19] and long tails 20], which are conneted to the appearance of a dressing cloud around the bare particle. The dressed unstable state defined through $\Lambda$ has a real average energy and gives an uncertainty relation between the lifetime and energy (see also 21]). Similar considerations can be applied in classical mechanics [22].

The present paper is organized as follows. In Secs. III - III we introduce the Friedrichs model and we discuss the equations of motion of the bare particle. In the subsequent Sections we study the evolution of renormalized (dressed) particle variables. We consider first (Sec. IV]) the integrable case where the spectrum of the field is discrete. We can then define the renormalized variables through the unitary transformation $U$. In the continuous spectrum limit the system becomes nonintegrable. In Sec. $[\nabla$ as a first step to introduce $\Lambda$, we extend the renormalized particle modes in the discrete case to the decaying "Gamow" modes in the nonintegrable case. In Sec. VI] we construct $\Lambda$. In Sec. VII] we show the correspondence between the solution of Langevin equation (with Gaussian white noise) and the $\Lambda$ transformed variables. Finally, in Sec. VIII we derive a Fokker-Planck equation for the $\Lambda$ transformed distribution function. Details of calculations are given in the Appendices.

\section{THE CLASSICAL FRIEDRICHS MODEL}

We consider a classical system consisting of a harmonic oscillator coupled to a classical scalar field in onedimensional space. A quantum version of this model has been studied by Friedrichs [2], among others.

We write the Hamiltonian of the system in terms of the bare oscillator and field modes $a_{1}$ and $a_{k}$,

$$
H=\omega_{1} a_{1}^{*} a_{1}+\sum_{k} \omega_{k} a_{k}^{*} a_{k}+\lambda \sum_{k} \bar{V}_{k}\left(a_{1}^{*} a_{k}+a_{1} a_{k}^{*}\right),
$$

with a given constant frequency $\omega_{1}>0$ for the harmonic oscillator (particle), $c=1$ for the speed of light, and $\omega_{k}=|k|$ for the field. When $\lambda$ is small we can treat the interaction potential as a perturbation. We assume the system is in a one-dimensional box of size $L$ with periodic boundary conditions. Then the spectrum of the field is discrete, i.e., $k=2 \pi j / L$ where $j$ is an integer. We assume that

$$
\omega_{1} \neq \omega_{k}, \quad \text { for all } k
$$

The volume dependence of the interaction $V_{k}$ is given by

$$
\bar{V}_{k}=\sqrt{\frac{2 \pi}{L}} \bar{v}_{k}
$$

where $\bar{v}_{k}=O(1)$. We assume that $\bar{v}_{k}$ is real and even: $\bar{v}_{k}=\bar{v}_{-k}$. Furthermore, we assume that for small $k$

$$
\bar{v}_{k} \sim \omega_{k}^{1 / 2} .
$$


An example is the Drude-Ullersma form [7]:

$$
\bar{v}_{k}=\frac{\omega_{k}^{1 / 2}}{1+\omega_{k}^{2} / \omega_{M}^{2}},
$$

where $\omega_{M}$ is the cutoff frequency of the bath.

To deal with the continuous spectrum of the field we take the limit $L \rightarrow \infty$. In this limit we have

$$
\frac{2 \pi}{L} \sum_{k} \rightarrow \int d k, \quad \frac{L}{2 \pi} \delta_{k, 0} \rightarrow \delta(k) .
$$

We will often use the summation sign with the understanding that we replace it by an integral in the limit $L \rightarrow \infty$.

The bare modes $a_{1}, a_{k}$ satisfy the Poisson bracket relation

$$
i\left\{a_{\alpha}, a_{\beta}^{*}\right\}=\delta_{\alpha \beta}
$$

where

$$
i\{f, g\}=\sum_{r}\left[\frac{\partial f}{\partial a_{r}} \frac{\partial g}{\partial a_{r}^{*}}-\frac{\partial g}{\partial a_{r}} \frac{\partial f}{\partial a_{r}^{*}}\right]
$$

[the sum includes the discrete index $r=1$ as well as the running index $r=k]$. The bare modes are related to the position $x_{1}$ and the momentum $p_{1}$ of the particle as

$$
\begin{aligned}
& a_{1}=\sqrt{\frac{m \omega_{1}}{2}}\left(x_{1}+\frac{i p_{1}}{m \omega_{1}}\right), \\
& x_{1}=\frac{1}{\sqrt{2 m \omega_{1}}}\left(a_{1}+a_{1}^{*}\right), \\
& p_{1}=-i \sqrt{\frac{m \omega_{1}}{2}}\left(a_{1}-a_{1}^{*}\right)
\end{aligned}
$$

and to the field $\phi(x)$ and its conjugate field $\pi(x)$ as

$$
\begin{aligned}
& \phi(x)=\sum_{k}\left(\frac{1}{2 \omega_{k} L}\right)^{1 / 2}\left(a_{k} e^{i k x}+a_{k}^{*} e^{-i k x}\right), \\
& \pi(x)=-i \sum_{k}\left(\frac{\omega_{k}}{2 L}\right)^{1 / 2}\left(a_{k} e^{i k x}-a_{k}^{*} e^{-i k x}\right) .
\end{aligned}
$$

The field $\phi(x)$ corresponds to the transverse vector potential in electromagnetism, while $\pi(x)$ corresponds to the transverse displacement field. Our Hamiltonian can be seen as a simplified version of a classical dipole molecule interacting with a classical radiation field in the dipole approximation 23]. For simplicity we neglect the interactions proportional to $a_{1} a_{k}$ and $a_{1}^{*} a_{k}^{*}$, which correspond to "virtual processes" in quantum mechanics. This approximation corresponds to the so-called rotating wave approximation [4, 24]. If we incorporate the virtual processes, then we obtain the classical version of the Caldeira-Leggett model.

We note that we have an $\omega_{k}=\omega_{-k}$ degeneracy in our Hamiltonian. To avoid some complexity due to this degeneracy, we rewrite our Hamiltonian in terms of new bare modes as 22]

$$
H=\omega_{1} q_{1}^{*} q_{1}+\sum_{k} \omega_{k} q_{k}^{*} q_{k}+\lambda \sum_{k} V_{k}\left(q_{1}^{*} q_{k}+q_{1} q_{k}^{*}\right),
$$

where

$$
\begin{aligned}
q_{1} \equiv a_{1}, \quad q_{k} \equiv \begin{cases}\left(a_{k}+a_{-k}\right) / \sqrt{2}, & \text { for } k>0, \\
\left(a_{k}-a_{-k}\right) / \sqrt{2}, & \text { for } k \leq 0,\end{cases} \\
V_{k} \equiv \begin{cases}\sqrt{2} \bar{V}_{k}, & \text { for } k>0 \\
0, & \text { for } k \leq 0,\end{cases} \\
v_{k}=\sqrt{\frac{L}{2 \pi}} V_{k} .
\end{aligned}
$$

In this form the mode $q_{k}$ with negative $k$ argument is completely decoupled from the other degrees of freedom. The new bare modes also satisfy equations (15), (16).

In the subsequent sections we will use the following notations. We define action and angle variables $J_{s}, \alpha_{s}$ through the relation

$$
q_{s}=\sqrt{J_{s}} e^{-i \alpha_{s}}, \quad s=1, k
$$

We define $\Gamma$ as the set of all modes,

$$
\Gamma \equiv\left(q_{1}, q_{1}^{*}, \ldots, q_{k}, q_{k}^{*}, \ldots\right)
$$

and $\Gamma_{s}=\left(q_{s}, q_{s}^{*}\right)$, with $s=1, k$, as the set of particle or field modes. We will also denote $\Gamma_{f}$ as the set of all field modes $\Gamma_{f}=\left\{\Gamma_{k}\right\}$. We use the notation $d \Gamma$ for the phase space volume element and $d \Gamma_{1}, d \Gamma_{f}$ for the particle and field components of $d \Gamma$, respectively

$$
\begin{aligned}
& d \Gamma=d \Gamma_{1} d \Gamma_{f} \\
& d \Gamma_{1}=d J_{1} d \alpha_{1}, \quad d \Gamma_{f}=\prod_{k} d J_{k} d \alpha_{k} .
\end{aligned}
$$

We define as well

$$
\begin{aligned}
& \delta\left(\Gamma-\Gamma^{\prime}\right) \\
& \equiv \delta\left(J_{1}-J_{1}^{\prime}\right) \delta\left(\alpha_{1}-\alpha_{1}^{\prime}\right) \prod_{k} \delta\left(J_{k}-J_{k}^{\prime}\right) \delta\left(\alpha_{k}-\alpha_{k}^{\prime}\right) .
\end{aligned}
$$

We consider ensemble averages as inner products:

$$
\langle F\rangle=\langle\langle F \mid \rho\rangle\rangle=\int d \Gamma F(\Gamma)^{*} \rho(\Gamma) .
$$

For an operator $O$ the Hermitian conjugate is defined by

$$
\langle\langle F \mid O \rho\rangle\rangle=\left\langle\left\langle\rho \mid O^{\dagger} F\right\rangle\right\rangle^{*} .
$$

As mentioned in the Introduction, for our model we can have both integrable ( $L$ finite) and nonintegrable cases $(L \rightarrow \infty)$. In the first case there are no resonances (see Eq. (10)) and as we will see, the the system is integrable in the sense of Poincaré. In the second case the system can become nonintegrable in Poincaré's sense, due to the emergence of the resonance $\omega_{1}=\omega_{k}$ between the frequencies of the particle and the field. This distinction is essential in our construction of dressed particle modes. Before coming to this, we will briefly consider the equations of motion for the bare modes. 


\section{EQUATIONS OF MOTION OF THE BARE PARTICLE MODES}

The dynamical equations of motion of an oscillator coupled to a field have been studied by many authors, mainly using the quantum Caldeira-Leggett model 3, 4, 5, 6, 7]. Here we will write the equations for the Friedrichs model. In contrast to the phenomenological equations describing Brownian motion [25], these equations have memory terms (i.e. they have time-dependent damping and diffusion coefficients), and the time evolution they generate forms a group, since they are equivalent to Hamiltonian dynamics.

\section{A. Non-Markovian Langevin equation}

Starting from the Hamiltonian equations

$$
q_{s}(t)=\exp \left(i L_{H} t\right) q_{s}(0) \Rightarrow \dot{q}_{s}(t)=i L_{H} q_{s}(t)
$$

we can obtain the exact time evolution of the modes $q_{r}$ as

$$
q_{s}(t)=\sum_{r} f_{s r}(t) q_{r}(0)
$$

where $f_{r s}(t)$ are complex functions (see Appendix A.

We will focus our attention on the particle modes:

$$
\begin{aligned}
& q_{1}(t)=f_{11}(t) q_{1}(0)+\sum_{k} f_{1 k}(t) q_{k}(0), \\
& \dot{q}_{1}(t)=\dot{f}_{11}(t) q_{1}(0)+\sum_{k} \dot{f}_{1 k}(t) q_{k}(0) .
\end{aligned}
$$

Solving for $q_{1}(0)$ in the first equation and replacing the result in the second equation we get

$$
\dot{q}_{1}(t)=-i z_{1}(t) q_{1}(t)+R(t)
$$

where

$$
\begin{aligned}
z_{1}(t) & =i \frac{\partial}{\partial t} \ln f_{11}(t), \\
R(t) & =\sum_{k} h_{k}(t) q_{k}(0), \\
h_{k}(t) & =\dot{f}_{1 k}(t)+i z_{1}(t) f_{1 k}(t) .
\end{aligned}
$$

Eq. (34) is a non-Markovian equation, because of the time dependence of the coefficients. The function $z_{1}(t) \equiv$ $\tilde{\omega}_{1}(t)-i \gamma(t)$ gives the instantaneous frequency $\tilde{\omega}_{1}(t)$ and damping rate $\gamma(t)$ of the oscillator [we note that damping appears only in the non-integrable case]. $R(t)$ is an erratic function, since it depends on the initial states of all the field modes $q_{k}(0)$ (see Appendix $\mathrm{B}$ ). It plays the role of noise. In general, this is colored noise, as the function $R(t)$ has memory in the auto-correlation,

$$
\left\langle R^{*}(t) R\left(t^{\prime}\right)\right\rangle \neq 0 \text { for } t \neq t^{\prime},
$$

where \langle\rangle means ensemble average.

\section{B. Non-Markovian Fokker-Planck equation}

We can also derive a non-Markovian equation for the particle distribution function

$$
\rho_{1}\left(\Gamma_{1}, t\right) \equiv \int d \Gamma_{f} \rho(\Gamma, t)
$$

which allows us to calculate averages of functions $G\left(\Gamma_{1}\right)$ depending only on the particle modes. We assume that $G\left(\Gamma_{1}\right)$ is a smooth real function of $\Gamma_{1}$ that vanishes at $\left|q_{1}\right|=\infty$ and is expandable in the infinite series

$$
G\left(q_{1}, q_{1}^{*}\right)=\sum_{m=0}^{\infty} \sum_{n=0}^{\infty} G_{m n} q_{1}^{* m} q_{1}^{n}
$$

We assume as well that $\rho(\Gamma, t)$ is factorized at $t=0$ into independent particle and field mode functions and that the field distributions depend only on the actions. In other words, we have

$$
\rho(\Gamma, 0)=\rho_{1}\left(\Gamma_{1}, 0\right) \prod_{k} \rho_{k}\left(J_{k}\right) .
$$

Our final assumption is that the volume of the system is large, so we neglect terms of order $1 / L$. This approximation becomes exact in the continuous spectrum limit $L \rightarrow \infty$, i.e., in the nonintegrable case. We consider the extensive case discussed in the Introduction.

Based on Eq. (34) we then obtain the non-Markovian equation (see Appendix C)

$$
\begin{aligned}
\frac{\partial}{\partial t} \rho_{1}\left(\Gamma_{1}, t\right) & =\left\{i z_{1}(t) \frac{\partial}{\partial q_{1}} q_{1}-i z_{1}^{*}(t) \frac{\partial}{\partial q_{1}^{*}} q_{1}^{*}\right. \\
& \left.+D(t) \frac{\partial^{2}}{\partial q_{1} \partial q_{1}^{*}}\right\} \rho_{1}\left(\Gamma_{1}, t\right),
\end{aligned}
$$

where

$$
D(t)=\sum_{k}\left[-i z_{1}^{*}(t)+i z_{1}(t)+\frac{\partial}{\partial t}\right]\left|f_{1 k}(t)\right|^{2}\left\langle J_{k}\right\rangle .
$$

Eq. (42) is of the Fokker-Planck type, but with time dependent coefficients.

The equations derived in this section are reminiscent of the phenomenological equations for Brownian motion. However, the phenomenological equations have quite important differences: they are Markovian, they break time-symmetry and they describe stochastic processes. One can derive the phenomenological equations using approximations, such as the Markovian approximation. This is shown in Appendix D

In the rest of the paper we will study the dynamical evolution of renormalized modes. In contrast to the bare modes, the renormalized modes obey exact equations having the same evolution as the phenomenological equations. As a preparation, we first consider the integrable case. 


\section{UNITARY TRANSFORMATION FOR INTEGRABLE CASE}

In this Section, we present the properties of the canonical transformation $U$ that diagonalizes the Hamiltonian in the discrete spectrum case, when the size of the box $L$ is finite. Later we will extend $U$ to $\Lambda$ through analytic continuation, for $L \rightarrow \infty$. In the integrable case we can find renormalized modes $\bar{Q}_{s}, \bar{Q}_{s}^{*}$ that diagonalize the Hamiltonian through $U$. The new modes are related to the bare modes as

$$
\bar{Q}_{s}=U^{\dagger} q_{s} \text { for } s=1, k .
$$

in one-to-one correspondence. The operator $U$ is unitary $U^{-1}=U^{\dagger}$.

The Hamiltonian is diagonalized as

$$
H=\sum_{s} \bar{\omega}_{s} \bar{Q}_{s}^{*} \bar{Q}_{s}
$$

where $\bar{\omega}_{\alpha}$ are renormalized frequencies.

The new modes satisfy the Poisson bracket relation

$$
i\left\{\bar{Q}_{r}, \bar{Q}_{s}^{*}\right\}=\delta_{r s} .
$$

Since the interaction is bilinear in the bare modes, the new modes can be found explicitly through a linear superposition of the bare modes 22]. For the particle we obtain, from the equation $i\left\{H, \bar{Q}_{1}\right\}=-\bar{\omega}_{1} \bar{Q}_{1}$,

$$
\bar{Q}_{1}=\bar{N}_{1}^{1 / 2}\left(q_{1}+\lambda \sum_{k} \bar{c}_{k} q_{k}\right)
$$

where

$$
\begin{aligned}
\bar{c}_{k} & \equiv \frac{V_{k}}{\bar{\omega}_{1}-\omega_{k}} \\
\bar{N}_{1} & \equiv(1+\bar{\xi})^{-1}, \quad \bar{\xi} \equiv \lambda^{2} \sum_{k} \bar{c}_{k}^{2} .
\end{aligned}
$$

The renormalized frequency $\bar{\omega}_{1}$ is given by the root of the equation

$$
\eta\left(\bar{\omega}_{1}\right)=0, \quad \eta(z) \equiv z-\omega_{1}-\sum_{k^{\prime}} \frac{\lambda^{2}\left|V_{k^{\prime}}\right|^{2}}{z-\omega_{k^{\prime}}}
$$

that reduces to $\omega_{1}$ when $\lambda=0$. For the field modes one can also find explicit forms (see Appendix A).

The perturbation expansion of Eq. (47) yields

$$
\bar{Q}_{1}=U^{\dagger} q_{1}=q_{1}+\sum_{k} \frac{\lambda V_{k}}{\omega_{1}-\omega_{k}} q_{k}+O\left(\lambda^{2}\right)
$$

When the spectrum is discrete, the denominator never vanishes; each term in the perturbation series is finite. This implies integrability in the sense of Poincaré: $U$ can be constructed by a perturbation series in powers of $\lambda^{n}$ with $n \geq 0$ integer. In other words, $U$ is analytic at $\lambda=0$.
Since the transformation $U$ is canonical, it is distributive with respect to multiplication

$$
U^{\dagger} q_{r} q_{s}^{*}=\left[U^{\dagger} q_{r}\right]\left[U^{\dagger} q_{s}^{*}\right]=\bar{Q}_{r} \bar{Q}_{s}^{*}
$$

Hence we have

$$
U H=U\left[\sum_{s} \bar{\omega}_{s} \bar{Q}_{s}^{*} \bar{Q}_{s}\right]=\sum_{s} \bar{\omega}_{s} q_{s}^{*} q_{s}=\bar{H}_{0}
$$

The transformed Hamiltonian $U H$ has the same form of the unperturbed Hamiltonian $H_{0}$, with renormalized frequencies.

The canonical transformation can also be introduced on the level of statistical ensembles $\rho$, as shown in the Introduction. In Eq. (3) we have

$$
\begin{aligned}
& \bar{L}_{0} \bar{\rho}=i U\{H, \rho\}=i\{U H, U \rho\} \\
& =\left[\sum_{s} \bar{\omega}_{s}\left(q_{s}^{*} \frac{\partial}{\partial q_{s}^{*}}-q_{s} \frac{\partial}{\partial q_{s}}\right)\right] U \rho
\end{aligned}
$$

where in the second equality we used Eq. (53) and the property of preservation of the Poisson bracket by canonical transformations 26. Hence the transformed Liouvillian $\bar{L}_{0}$ does not contain any interaction terms. Ensemble averages over this transformed density function $\bar{\rho}$ can thus be easily calculated. For example for

$$
i \frac{\partial}{\partial t}\left\langle\left\langle x_{1}|U| \rho\right\rangle\right\rangle=i \frac{\partial}{\partial t}\left\langle\bar{x}_{1}\right\rangle
$$

and similarly for $\left\langle\bar{p}_{1}\right\rangle$ we get, after substituting Eq. (18) and integrating by parts,

$$
\frac{\partial}{\partial t}\left\langle\bar{x}_{1}\right\rangle=\frac{1}{\bar{m}}\left\langle\bar{p}_{1}\right\rangle, \quad \frac{\partial}{\partial t}\left\langle\bar{p}_{1}\right\rangle=-\bar{m} \bar{\omega}_{1}^{2}\left\langle\bar{x}_{1}\right\rangle .
$$

These are the equations for the free harmonic oscillator (with renormalized frequency $\bar{\omega}_{1}$ and renormalized mass $\left.\bar{m}=m \omega_{1} / \bar{\omega}_{1}\right)$. The interaction with the field is eliminated.

Note that the normal modes are eigenfunctions of the Liouvillian $\bar{L}_{0}$,

$$
\bar{L}_{0} q_{1}=-\bar{\omega}_{1} q_{1}, \quad \bar{L}_{0} q_{1}^{*}=\bar{\omega}_{1} q_{1}^{*} .
$$

This leads to

$$
L_{H} \bar{Q}_{1}=-\bar{\omega}_{1} \bar{Q}_{1}, \quad L_{H} \bar{Q}_{1}^{*}=\bar{\omega}_{1} \bar{Q}_{1}^{*}
$$

For products of modes we have

$$
\begin{aligned}
\bar{L}_{0} q_{1}^{* m} q_{1}^{n} & =\left[(m-n) \bar{\omega}_{1}\right] q_{1}^{* m} q_{1}^{n}, \\
L_{H} \bar{Q}_{1}^{* m} \bar{Q}_{1}^{n} & =\left[(m-n) \bar{\omega}_{1}\right] \bar{Q}_{1}^{* m} \bar{Q}_{1}^{n} .
\end{aligned}
$$

Finally, we note that from distributive property Eq. (52) we have

$$
U^{\dagger} q_{1}^{* m} q_{1}^{n}=\left(U^{\dagger} q_{1}^{* m}\right)\left(U^{\dagger} q_{1}^{n}\right)
$$




\section{NONINTEGRABLE CASE: GAMOW MODES}

Now we consider the continuous spectrum case, where the particle frequency $\omega_{1}$ is inside the range of the continuous spectrum $\omega_{k}$. In this case, by analytic continuation of $\bar{Q}_{1}$ and $\bar{Q}_{1}^{*}$ we can get new modes which are eigenfunctions of the Liouvillian with complex eigenvalues. These modes are called Gamow modes. Gamow states have been previously introduced in quantum mechanics to study unstable states [27]- 32]. In classical mechanics, Gamow modes have been introduced in Ref. 22]. In this Section we present the main properties of Gamow modes, which will be used for the construction of $\Lambda$.

When we go to the continuous limit we restrict the strength of the coupling constant $\lambda$ so that

$$
\int d k \frac{\lambda^{2}\left|v_{k}\right|^{2}}{\omega_{k}}<\omega_{1}
$$

Then the harmonic oscillator becomes unstable. In this case we have radiation damping. If Eq. 61] is not satisfied, then we go outside the range of applicability of the "rotating wave approximation" (see comment after Eq. (20) as the Hamiltonian becomes not bounded from below, and gives no radiation damping [33].

In the continuous spectrum case, divergences appear in the construction of $U$, due to resonances. For example, the denominator in Eq. (51) may now vanish at the Poincaré resonance $\omega_{1}=\omega_{k}$. We have a divergence in the perturbation expansion in $\lambda$. To deal with this divergence, we regularize the denominator by adding an infinitesimal $\pm i \epsilon$. Then we get

$$
Q_{1}=q_{1}+\sum_{k} \frac{\lambda V_{k}}{\omega_{1}-\omega_{k} \pm i \epsilon} q_{k}+O\left(\lambda^{2}\right) .
$$

In the continuous limit the summation goes to an integral. We take the limit $L \rightarrow \infty$ first and $\epsilon \rightarrow \infty$ later. Then the denominator can be interpreted as a distribution under the integration over $k$

$$
\frac{1}{\omega_{1}-\omega_{k} \pm i \epsilon} \rightarrow \mathcal{P} \frac{1}{\omega_{1}-\omega_{k}} \mp i \pi \delta\left(\omega_{1}-\omega_{k}\right)
$$

where $\mathcal{P}$ means principal part.

The introduction of $i \epsilon$ in the continuous limit is related to a change of the physical situation. In the discrete case the boundaries of the system cause periodicity in the motion of the particle and the field. In contrast, in the continuous case the boundaries play no role. In the continuous limit we can have damping of the particle, as the field emitted from the particle goes away and never comes back. And we can have Brownian motion, due to the interaction with the continuous set of field modes. The continuous limit may be well approximated by a discrete system during time scales much shorter than the time scale for which the field goes across the boundaries.

In the continuous limit we can have damping of the particle either toward the future or toward the past. This corresponds to the existence of the two branches $\pm i \epsilon$ in Eq. (62). Breaking of time symmetry is connected to resonances [34].

As shown in 30], continuing the perturbation expansion (62) to all orders one obtains new renormalized modes (Gamow modes) associated with the complex frequency

$$
z_{1} \equiv \tilde{\omega}_{1}-i \gamma
$$

or its complex conjugate $z_{1}^{*}$. Here $\tilde{\omega}_{1}$ is the renormalized frequency of the particle, and $2 \gamma>0$ is the damping rate. The complex frequencies are solutions of the equation

$$
\eta^{ \pm}(\omega)=\omega-\omega_{1}-\int d k \frac{\lambda^{2} v_{k}^{2}}{\left(z-\omega_{k}\right)_{\omega}^{ \pm}}=0 .
$$

The $+(-)$ superscript indicates that the propagator is first evaluated on the upper (lower) half plane of $z$ and then analytically continued to $z=\omega$. by

The new modes for the $-i \epsilon$ branch in Eq. (62) are given

$$
\begin{aligned}
\tilde{Q}_{1} & =N_{1}^{1 / 2}\left[q_{1}+\lambda \sum_{k} c_{k} q_{k}\right], \\
c_{k} & =\frac{V_{k}}{\left(z-\omega_{k}\right)_{z_{1}}^{+}}, \quad N_{1}=\left(1+\lambda^{2} \sum_{k} c_{k}^{2}\right)^{-1}
\end{aligned}
$$

and its complex conjugate, satisfying

$$
L_{H} \tilde{Q}_{1}=-z_{1} \tilde{Q}_{1}, \quad L_{H} \tilde{Q}_{1}^{*}=z_{1}^{*} \tilde{Q}_{1}^{*} .
$$

The mode $\tilde{Q}_{1}^{*}$ decays for $t>0$ as

$$
e^{i L_{H} t} \tilde{Q}_{1}^{*}=e^{i z_{1}^{*} t} \tilde{Q}_{1}^{*}=e^{\left(i \tilde{\omega}_{1}-\gamma\right) t} \tilde{Q}_{1}^{*}
$$

(and similarly $\tilde{Q}_{1}$ ).

The modes for the $+i \epsilon$ branch are given by

$$
Q_{1}^{*}=N_{1}^{1 / 2}\left[q_{1}^{*}+\lambda \sum_{k} c_{k} q_{k}^{*}\right]
$$

and its complex conjugate, satisfying

$$
L_{H} Q_{1}^{*}=z_{1} Q_{1}^{*}, \quad L_{H} Q_{1}=-z_{1}^{*} Q_{1} .
$$

These modes decay for $t<0$.

The modes we have introduced have quite different properties from the usual canonical variables. Their Poisson brackets vanish

$$
i\left\{Q_{1}, Q_{1}^{*}\right\}=i\left\{\tilde{Q}_{1}, \tilde{Q}_{1}^{*}\right\}=0 .
$$

However the modes $\tilde{Q}_{1}$ and $Q_{1}^{*}$ are duals; they form a generalized canonical pair

$$
i\left\{\tilde{Q}_{1}, Q_{1}^{*}\right\}=1 .
$$

This algebra corresponds to an extension of the usual Lie algebra including dissipation. An analogue of this algebra has been previously studied in quantum mechanics [27][32], in terms of non-Hilbertian bras and kets. 


\section{THE $\Lambda$ TRANSFORMATION}

Using the above results we now introduce $\Lambda$. In this paper we will restrict the action of $\Lambda$ to products of particle modes of the form $q_{1}^{* m} q_{1}^{n}$. This will be enough to calculate renormalized functions of the particle variables (expandable in monomials), which will lead us to the Langevin and Fokker-Planck equations. The action of $\Lambda$ on more general functions, including field modes will be considered elsewhere (see also [15, 22]).

\section{A. Defining $\Lambda$ through its action on particle modes}

First recall that in the integrable case the renormalized particle modes are related to the original modes as

$$
\bar{Q}_{1}=U^{\dagger} q_{1}, \quad \bar{Q}_{1}^{*}=U^{\dagger} q_{1}^{*}
$$

For products of modes we have as well the relation (60). In the continuous spectrum limit we come to the nonintegrable case. As seen in the previous Section, we eliminate Poincaré divergences in single renormalized particle modes by analytic continuation of frequencies to the complex plane (i.e. $\bar{\omega}_{1}$ goes to $z_{1}$ ) leading to Gamow modes. There are two branches for the continuation, namely

$$
\bar{Q}_{1} \Rightarrow\left\{\begin{array}{l}
\tilde{Q}_{1} \\
Q_{1}
\end{array}\right.
$$

Corresponding to these extensions, we introduce $\Lambda$, the extension of $U$ in Eq. (74),

$$
\begin{aligned}
& \tilde{Q}_{1}=\Lambda^{\dagger} q_{1}, \quad \tilde{Q}_{1}^{*}=\Lambda^{\dagger} q_{1}^{*} \\
& Q_{1}=\Lambda^{-1} q_{1}, \quad Q_{1}^{*}=\Lambda^{-1} q_{1}^{*}
\end{aligned}
$$

These relations partially define $\Lambda$, by its action on single particle modes (a more complete definition is given below). This definition satisfies the requirements (1), (3) and (4) given in the Introduction. We will comment on the remaining requirements (2) and (5) below. Note that $\Lambda^{\dagger} \neq \Lambda^{-1}$ is not unitary. Instead, it is "star-unitary,"

$$
\Lambda^{-1}=\Lambda^{\star}
$$

In our case, where we restrict the action of $\Lambda$ to particle modes, star conjugation has a simple meaning. It simply means taking hermitian conjugation and changing $i \epsilon \Rightarrow$ $-i \epsilon$, so we have, e.g., $\left[\Lambda^{\star}(i \epsilon)\right] q_{1}=\left[\Lambda^{\dagger}(-i \epsilon)\right] q_{1}$. For the general definition of star conjugation, see [15, 30] and references therein.

Due to star-unitarity, the existence of the starconjugate transformation $\Lambda^{\star}$ guarantees the existence of the inverse $\Lambda^{-1}$.

As mentioned above we are interested not only in the renormalized modes, but also the renormalized products of modes,

$$
\Lambda^{\dagger} q_{1}^{* m} q_{1}^{n}, \quad \Lambda^{-1} q_{1}^{* m} q_{1}^{n}
$$

For the integrable case, renormalized products of modes can be easily calculated thanks to the distributive property (60). However, as shown below, for the nonintegrable case products of Gamow modes give new Poincaré divergences. Hence, due to the requirement (4) stated in the Introduction, the $\Lambda$ transformation has to be non-distributive. This means that we still have to define the action of $\Lambda$ on products of particle modes.

Let us first consider the transformed product $\Lambda^{\dagger} q_{1}^{*} q_{1}$. Later we will generalize this to obtain the expressions (78). If $\Lambda^{\dagger}$ were distributive, $\Lambda^{\dagger} q_{1}^{*} q_{1}$ could be expressed as the product $\tilde{Q}_{1}^{*} \tilde{Q}_{1}=\left(\Lambda^{\dagger} q_{1}^{*}\right)\left(\Lambda^{\dagger} q_{1}\right)$. However, as we show now, this expression gives Poincaré divergences in the thermodynamic limit. We have

$$
\begin{aligned}
& \tilde{Q}_{1}^{*} \tilde{Q}_{1} \\
= & \left|N_{1}\right|\left(q_{1}^{*}+\lambda \sum_{k} c_{k}^{*} q_{k}^{*}\right)\left(q_{1}+\lambda \sum_{k} c_{k} q_{k}\right) \\
= & \left|N_{1}\right|\left(q_{1}^{*} q_{1}+\lambda q_{1}^{*} \sum_{k} c_{k} q_{k}+\lambda q_{1} \sum_{k} c_{k}^{*} q_{k}^{*}\right. \\
+ & \left.\lambda^{2} \sum_{k, k^{\prime}}^{\prime} c_{k}^{*} c_{k^{\prime}} q_{k}^{*} q_{k^{\prime}}+\lambda^{2} \sum_{k}\left|c_{k}\right|^{2} q_{k}^{*} q_{k}\right) .
\end{aligned}
$$

where the prime in the summation means $k \neq k^{\prime}$. Going to the continuous limit and taking the ensemble average with an ensemble $\rho$ the last term becomes

$$
\sum_{k}\left|c_{k}\right|^{2}\left\langle q_{k}^{*} q_{k}\right\rangle \rightarrow \int d k\left|\frac{\lambda v_{k}}{\left(z-\omega_{k}\right)_{z_{1}}^{+}}\right|^{2}\left\langle J_{k}\right\rangle
$$

where $\left\langle J_{k}\right\rangle=\left\langle\left\langle q_{k}^{*} q_{k} \mid \rho\right\rangle\right\rangle$. This term has a non-vanishing finite value in the limit $L \rightarrow \infty$ if Eq. (7) is satisfied. Furthermore, if the ensemble $\rho$ belongs to the class of ensembles with $\delta$-function singularities in the wave number $k$, then Eq. (80) is non-negligible as compared to the average of the $q_{k}^{*} q_{k^{\prime}}$ term in Eq. (79). For this class of ensembles the point contribution $k=k^{\prime}$ is as important as the integration over $k^{\prime}$ [13, 14, 35]:

$$
\sum_{k^{\prime}}\left\langle q_{k}^{*} q_{k^{\prime}}\right\rangle \sim\left\langle J_{k}\right\rangle \sim O\left(L^{0}\right) .
$$

(see Appendix E). This type of ensembles with $\delta$-function singularities is by no means atypical. An example of this class of ensembles is the Gibbs distribution. For ensembles in this class, we have well defined intensive and extensive variables in the thermodynamic limit [35].

To lowest order we have in Eq. (80),

$$
\frac{\lambda v_{k}}{\left(z-\omega_{k}\right)_{z_{1}}^{+}}=\frac{\lambda v_{k}}{\omega_{1}-\omega_{k}+i \epsilon}+O\left(\lambda^{3}\right)
$$

which leads to

$$
\begin{aligned}
\left|\frac{\lambda v_{k}}{\left(z-\omega_{k}\right)_{z_{1}}^{+}}\right|^{2} & =\frac{\lambda^{2} v_{k}^{2}}{\left|\omega_{1}-\omega_{k}+i \epsilon\right|^{2}}+O\left(\lambda^{4}\right) \\
& =\frac{\pi}{\epsilon} \lambda^{2} v_{k}^{2} \delta\left(\omega_{1}-\omega_{k}\right)+O\left(\lambda^{4}\right) \rightarrow \infty
\end{aligned}
$$


This diverges when $\epsilon \rightarrow 0$. Hence Eq. (80) is nonanalytic at $\lambda=0$ due to the resonance at $\omega_{1}=\omega_{k}$. We have Poincaré divergence in the perturbation series of $\left(\Lambda^{\dagger} q_{1}^{*}\right)\left(\Lambda^{\dagger} q_{1}\right)$.

We note that when the energy of the field is nonextensive, we have $\left\langle J_{k}\right\rangle \sim O(1 / L)$. The energy density goes to zero in the infinite volume limit. In this case the appearance of the Poincaré divergence in Eq. (80) has no effect on the particle.

For quantum mechanics the situation is different. We can have fluctuations even in non-extensive situations [15] due to vacuum effects. For example we obtain, for a two-level atom, an energy fluctuation of the dressed excited state which is of the order of the decay rate. This gives an uncertainty relation between energy and lifetime.

Coming back to our main discussion, we conclude that $\Lambda^{\dagger} q_{1}^{*} q_{1}$ cannot be expressed as the product Eq. (79) since $\Lambda$ is, by definition, analytic in the coupling constant. To make this transformed product analytic, we make the replacement

$$
\lambda^{2} \sum_{k}\left|c_{k}\right|^{2} q_{k}^{*} q_{k} \Rightarrow \lambda^{2} \sum_{k} \xi_{k} q_{k}^{*} q_{k}
$$

where $\xi_{k}$ is a suitable analytic function. Due to the requirements on $\Lambda$ stated in the Introduction this function is not quite arbitrary. Indeed, in the integrable case the term $q_{k}^{*} q_{k}$ would appear in $U^{\dagger} q_{1}^{*} q_{1}$ as $\lambda^{2} \sum_{k} \bar{c}_{k}^{2} q_{k}^{*} q_{k}$ (see Eq. (47)). In the nonintegrable case $\bar{c}_{k}$ is extended to $c_{k}$ or $c_{k}^{*}$, and becomes complex. Taking into account the requirements (1), (3) and (4) in the Introduction we conclude that a suitable extension of $\bar{c}_{k}^{2}$ to the nonintegrable case is the linear superposition

$$
\xi_{k}=r c_{k}^{2}+\text { c.c. }, \quad r+r^{*}=1
$$

where $r$ is a complex constant to be determined. The relation $r+r^{*}=1$ is the simplest relation which guarantees $\xi_{k}$ reduces to $\bar{c}_{k}^{2}$ in the integrable case [see also the comments below Eq. [F13].

So we have [4]

$$
\Lambda^{\dagger} q_{1}^{*} q_{1}=\tilde{Q}_{1}^{*} \tilde{Q}_{1}+\sum_{k} b_{k} q_{k}^{*} q_{k}
$$

where

$$
b_{k}=\lambda^{2}\left|N_{1}\right|\left(-\left|c_{k}\right|^{2}+\xi_{k}\right) .
$$

As shown in Appendix [Fusing the requirement (2) we obtain

$$
r=\frac{\exp (-i a / 2)}{2 \cos (a / 2)}, \quad N_{1}=\left|N_{1}\right| \exp (-i a)
$$

giving a concrete form of $\Lambda$ in Eq. [86). By including the term $b_{k}$ in Eq. 866 we have removed the Poincaré divergence in the product of Gamow modes. As a consequence,

$$
\Lambda^{\dagger} q_{1}^{*} q_{1} \neq\left(\Lambda^{\dagger} q_{1}^{*}\right)\left(\Lambda^{\dagger} q_{1}\right)
$$

This shows the non-distributive property of $\Lambda$.

For weak coupling the approximate value of $b_{k}$ is given by [15],

$$
b_{k} \approx \frac{2 \pi}{L} \frac{\lambda^{2} v_{k}^{2} \gamma^{2}}{\left[\left(\omega_{k}-\tilde{\omega}_{1}\right)^{2}+\gamma^{2}\right]^{2}} .
$$

This has a sharp peak at $\omega_{k}=\tilde{\omega}_{1}$ with a width $\gamma$. It corresponds to the line shape of emission and absorption of the field by the renormalized particle.

To find more general transformed products $\Lambda^{\dagger} q_{1}^{* m} q_{1}^{n}$, we apply the same logic that led to Eq. (86). Whenever $\left|c_{k}\right|^{2}$ appears in $\tilde{Q}_{1}^{* m} \tilde{Q}_{1}^{n}$, we replace it with $\xi_{k}$. This leads to (see Appendix G]).

$$
\begin{aligned}
& \Lambda^{\dagger} q_{1}^{* m} q_{1}^{n} \\
& =\sum_{a=0}^{m i n(m, n)} \frac{m ! n !}{(m-a) !(n-a) ! a !} \tilde{Q}_{1}^{* m-a} \tilde{Q}_{1}^{n-a} Y^{a}
\end{aligned}
$$

where $\min (m, n)$ is the smaller of $m, n$, and

$$
Y \equiv \sum_{k} b_{k} q_{k}^{*} q_{k}
$$

Note that $b_{k} \sim O(1 / L)$. Hence $Y \sim O\left(L^{0}\right)$ only if the field obeys the thermodynamic limit condition, Eq. (7). Otherwise $Y$ vanishes as $1 / L$ and $\Lambda^{\dagger}$ becomes distributive. Also, when there are no resonances, $z_{1}$ becomes real and both $b_{k}$ and $Y$ vanish. Then $\Lambda^{\dagger}$ reduces to $U^{\dagger}$ (see Eq. (60)). In short, both thermodynamic limit and resonances are necessary to obtain non distributivity of $\Lambda^{\dagger}$ in Eq. (91), which, as we will see in the next section, gives the fluctuations found in Brownian motion.

For $\Lambda^{-1} q_{1}^{* m} q_{1}^{n}$ we obtain the expression (91) with $\tilde{Q}_{1}, \tilde{Q}_{1}^{*}$ replaced by $Q_{1}, Q_{1}^{*}$, respectively.

\section{B. Obtaining closed Markovian equations}

The $\Lambda$ transformation we have presented satisfies all our requirements (1)-(4) stated in the Introduction. Now we show that $\Lambda$ also satisfies the requirement (5) namely, that $\Lambda$ gives closed Markovian equations. To obtain closed Markovian kinetic equations, we first operate $\Lambda$ on the Liouville equation, to obtain Eq. (5). Kinetic equations involve a projection (or a part) of the ensemble $\tilde{\rho}$. In order for $\Lambda$ to give closed kinetic of equations, we require that the transformed Liouvillian $\tilde{\theta}$ in Eq. (5) leaves subspaces corresponding to projections of $\tilde{\rho}$ invariant. We will represent these subspaces by projection operators $P^{(\nu)}$, which are orthogonal and complete in the domain of $\tilde{\theta}$,

$$
P^{(\mu)} P^{(\nu)}=P^{(\mu)} \delta_{\mu \nu}, \quad \sum_{\nu} P^{(\nu)}=1 .
$$

The invariance property of $\tilde{\theta}$ is

$$
P^{(\nu)} \tilde{\theta}=\tilde{\theta} P^{(\nu)} .
$$


Thanks to this commutation property, we obtain from Eq. (5) closed Markovian equations for the projections of $\tilde{\rho}$,

$$
i \frac{\partial}{\partial t} P^{(\nu)} \tilde{\rho}(t)=\tilde{\theta} P^{(\nu)} \tilde{\rho}(t)
$$

We choose $P^{(\nu)}$ as eigenprojectors of $L_{0}$. We have $L_{0} P^{(\nu)}=w^{(\nu)} P^{(\nu)}$ where $w^{(\nu)}$ are the eigenvalues. Then, for the integrable case the relation (94) is automatically satisfied, since in this case $\tilde{\theta}$ reduces to $\bar{L}_{0}$, the renormalized free Liouvillian with eigenprojectors $P^{(\nu)}$.

In the Friedrichs model the $P^{(\nu)}$ subspaces consist of monomials (or superposition of monomials) of field and particle modes. For example the monomials

$$
q_{1}^{*} q_{k}=P^{(1 k)} q_{1}^{*} q_{k}, \quad q_{1}^{*} q_{k} q_{l}^{*} q_{l}=P^{(1 k)} q_{1}^{*} q_{k} q_{l}^{*} q_{l}
$$

belong to the same subspace $P^{(1 k)}$ with eigenvalue $w^{(1 k)} \equiv \omega_{1}-\omega_{k}$.

One may introduce a Hilbert space structure for the eigenfunctions of $L_{0}$, including suitable normalization constants in the Segal-Bargmann representation 22]. We also note that $\Lambda^{\dagger}$ transformed variables $\Lambda^{\dagger} P^{(\nu)} A$ generate the kinetic equation (95) as we have $\left\langle\left\langle\Lambda^{\dagger} P^{(\nu)} A \mid \rho(t)\right\rangle\right\rangle=$ $\left\langle\left\langle A\left|P^{(\nu)}\right| \tilde{\rho}\right\rangle\right\rangle$.

Now we verify that the relation (94) is satisfied for the $\Lambda$ transformation we have constructed. We restrict ourselves to the components associated with the monomials in Eq. (78). These belong to eigenspaces of $L_{0}$ with eigenvalues $(m-n) \omega_{1}$. We denote the corresponding projectors as $P^{(m n)}$. Using Eq. (I4) in Appendix @ with $q_{1}^{\prime}=0$, we find

$$
\tilde{\theta}^{\dagger} q_{1}^{* m} q_{1}^{n}=\left[\left(m z_{1}^{*}-n z_{1}\right) q_{1}^{*} q_{1}-2 i \gamma m n Y\right] q_{1}^{* m-1} q_{1}^{n-1}
$$

and similarly

$$
\tilde{\theta} q_{1}^{* m} q_{1}^{n}=\left[\left(m z_{1}-n z_{1}^{*}\right) q_{1}^{*} q_{1}+2 i \gamma m n Y\right] q_{1}^{* m-1} q_{1}^{n-1} .
$$

Both the 1.h.s. and the r.h.s. of these two equations belong to the same eigenspace $P^{(m n)}$. This illustrates the statement that $\tilde{\theta}$ leaves the subspaces $P^{(\nu)}$ invariant, satisfying the requirement (5).

Due to the $Y$ term, $q_{1}^{* m} q_{1}^{n}$ are not eigenfunctions of $\tilde{\theta}$, so $P^{(m n)}$ is not an eigenprojector of $\tilde{\theta}$. This is quite natural, since the kinetic processes include both the decay of the particle modes (through emission of the field) and the absorption of field modes. These correspond to the first and second terms inside brackets in Eqs. (97), (98), respectively.

\section{C. П subdynamics}

We comment on the theory of $\Pi$ subdynamics, developed by the Brussels school [11]. We introduce the projectors

$$
\Pi^{(\nu)}=\Lambda^{-1} P^{(\nu)} \Lambda
$$

Similar to Eq. (93), they are orthogonal and complete. From Eq. (94) it follows that $\Pi^{(\nu)} L_{H}=L_{H} \Pi^{(\nu)}$. Hence these projectors define independent subspaces that follow independent, closed dynamics. The projectors themselves may be written in terms of generalized eigenstates of $L_{H}$, which give a complex spectral representation of this operator 14].

From the completeness relation of the $P^{(\nu)}$ or $\Pi^{(\nu)}$ projectors, we can recover the time evolution in the original variables as

$$
\rho(t)=\sum_{\nu} \Lambda^{-1} P^{(\nu)} \tilde{\rho}(t)=\sum_{\nu} \Pi^{(\nu)} \rho(t) .
$$

This shows that, as pointed out in the Introduction, dynamics is decomposed into a set of components obeying Markovian equations. In order to actually use Eq. (100) we need to know all the components $P^{(\nu)} \tilde{\rho}(t)$ (or equivalently, $\left.\Pi^{(\nu)} \rho(t)\right)$. At the present moment we have only obtained a restricted set of these. This is enough for our present goal. Rather than solving the original equations of motion (which can be done by other standard methods) our goal is to show that Brownian motion, Gaussian white noise and damping are part of dynamics seen in the $\Lambda$ representation.

The contruction of $\Lambda$ we have presented here is based on renormalized particle modes. A more general construction of $\Lambda$ starts with the commutation relation (94) together with the other requirements stated in the Introduction. The main idea is to associate a "degree of correlation" with each subspace $P^{(\nu)}$. Dynamics induces transitions among different $P^{(\nu)}$ subspaces. We have a "dynamics of correlations" [35]. This allows us to perform the regularization of denominators of $U$ in a systematic way, depending on types of transitions (from lower to higher correlations or vice versa), which leads to $\Lambda$. The interested reader can find a presentation of this formulation in Refs. 11, 15, 22].

\section{COMPARISON WITH THE PHENOMENOLOGICAL LANGEVIN EQUATION}

In this Section we discuss the relation between the solution of the phenomenological Langevin equation for the Friedrichs model and $\Lambda^{\dagger}$ transformed particle modes (i.e. dressed modes). We will focus on the $\Lambda^{\dagger}$ transformation, so that the transformed variables decay for $t>0$ [48] (see Eq. (69)). Remarkably, the Langevin equation and the dynamical equations for dressed modes have the same solution.

The phenomenological Langevin equation for the Brownian harmonic oscillator appropiate for the Friedrichs model has the form $(t>0)$

$$
\frac{d}{d t} \hat{q}_{1}(t)=-i \hat{z}_{1} \hat{q}_{1}(t)+\hat{R}(t)
$$

where $\hat{R}(t)$ is a complex noise source (see Eq. (34)). We use the hats to denote phenomenological variables. The 
complex coefficient $\hat{z}_{1} \equiv \hat{\omega}_{1}-i \hat{\gamma}$ gives the frequency and the damping rate of the oscillator. We assume $\hat{R}(t)$ has the following properties:

(1) $\hat{R}^{*}(t)$ and $\hat{R}\left(t^{\prime}\right)$ have the delta function (white noise) correlation.

$$
\left\langle\hat{R}^{*}(t) \hat{R}\left(t^{\prime}\right)\right\rangle=\hat{R}_{c}^{2} \delta\left(t-t^{\prime}\right)
$$

where \langle\rangle means noise average.

(2) $\hat{R}(t)$ has the Gaussian property

$$
\begin{aligned}
& \left\langle\hat{R}^{*}\left(t_{1}\right) \ldots \hat{R}^{*}\left(t_{m}\right) \hat{R}\left(t_{1}^{\prime}\right) \ldots \hat{R}\left(t_{n}^{\prime}\right)\right\rangle \\
& =\delta_{m n} \sum_{\text {all pairs }}\left\langle\hat{R}^{*}\left(t_{i_{1}}\right) \hat{R}\left(t_{j_{1}}^{\prime}\right)\right\rangle \cdots\left\langle\hat{R}^{*}\left(t_{i_{n}}\right) \hat{R}\left(t_{j_{n}}^{\prime}\right)\right\rangle .
\end{aligned}
$$

The noise constant $\hat{R}_{c}$ is determined in Appendix H using equipartition of energy and assuming the bath is at temperature $T$. The result is $\hat{R}_{c}^{2}=2 \hat{\gamma} k_{B} T / \hat{\omega}_{1}$.

Eq. (101) corresponds to the equations

$$
\begin{aligned}
& \frac{d}{d t} \hat{x}_{1}(t)=-\hat{\gamma} \hat{x}_{1}(t)+\frac{\hat{p}_{1}(t)}{\hat{m}}+A(t), \\
& \frac{d}{d t} \hat{p}_{1}(t)=-\hat{\gamma} \hat{p}_{1}(t)-\hat{m} \hat{\omega}_{1}^{2} \hat{x}_{1}(t)+B(t) .
\end{aligned}
$$

where $A(t)$ and $B(t)$ are independent Gaussian white noises [36]. These equations describe a damped harmonic oscillator with random momentum and force terms $A(t)$ and $B(t)$, respectively. The equations are symmetrical under rescaled position and momentum exchange, which is consistent with the same symmetry of the Hamiltonian.

The solution of Eq. (101) is given by

$$
\hat{q}_{1}(t)=\hat{q}_{1 a}(t)+\hat{q}_{1 r}(t),
$$

where

$$
\begin{aligned}
& \hat{q}_{1 a}(t) \equiv \hat{q}_{1}(0) e^{-i \hat{z}_{1} t}, \\
& \hat{q}_{1 r}(t) \equiv e^{-i \hat{z}_{1} t} \int_{0}^{t} d t^{\prime} \hat{R}\left(t^{\prime}\right) e^{i \hat{z}_{1} t^{\prime}} .
\end{aligned}
$$

The term $\hat{q}_{1 a}(t)$ describes the damped harmonic oscillator without noise, and the term $\hat{q}_{1 r}(t)$ describes the behavior due to the noise.

For later comparison we calculate the autocorrelation function $\left\langle\hat{q}_{1}^{* m}(t) \hat{q}_{1}^{n}(t)\right\rangle$. We have

$$
\begin{aligned}
& \left\langle\hat{q}_{q}^{* m}(t) \hat{q}_{1}^{n}(t)\right\rangle \\
& =\left\langle\left(\hat{q}_{1 a}^{*}(t)+\hat{q}_{1 r}^{*}(t)\right)^{m}\left(\hat{q}_{1 a}(t)+\hat{q}_{1 r}(t)\right)^{n}\right\rangle \\
& =\sum_{k=0}^{m} \sum_{l=0}^{n} \frac{m !}{(m-k) ! k !} \frac{n !}{(n-l) ! ! !} \\
& \times \hat{q}_{1 a}^{* m-k}(t) \hat{q}_{1 a}^{n-l}(t)\left\langle\hat{q}_{1 r}^{* k}(t) \hat{q}_{1 r}^{l}(t)\right\rangle .
\end{aligned}
$$

The quantity $\left\langle\hat{q}_{1 r}^{* k}(t) \hat{q}_{1 r}^{l}(t)\right\rangle$ is non-zero only when $k=$ $l$, as we can see from Eq. (103). Considering the fact that the number of sets of all possible pairs in $\left\langle\hat{R}^{*}\left(t_{1}\right) \ldots \hat{R}^{*}\left(t_{l}\right) \hat{R}\left(t_{1}^{\prime}\right) \ldots \hat{R}\left(t_{l}^{\prime}\right)\right\rangle$ is $l$ !, we have

$$
\begin{aligned}
& \left\langle\hat{q}_{1 r}^{* k}(t) \hat{q}_{1 r}^{l}(t)\right\rangle \\
& =l ! \delta_{k l}\left(\left\langlee^{i \hat{z}_{1}^{*} t} \int_{0}^{t} d t_{1} \hat{R}^{*}\left(t_{1}\right) e^{-i \hat{z}_{1}^{*} t_{1}}\right.\right. \\
& \left.\left.\times e^{-i \hat{z}_{1} t} \int_{0}^{t} d t_{2} \hat{R}\left(t_{2}\right) e^{i \hat{z}_{1} t_{2}}\right\rangle\right)^{l} \\
& =l ! \delta_{k l}\left(\frac{\hat{R}_{c}^{2}\left(1-e^{-2 \hat{\gamma} t}\right)}{2 \hat{\gamma}}\right)^{l} \\
& =l ! \delta_{k l}\left(\frac{k_{B} T}{\hat{\omega}_{1}}\right)^{l}\left(1-e^{-2 \hat{\gamma} t}\right)^{l} .
\end{aligned}
$$

Substituting Eq. (107) and Eq. (110) into Eq. (109), we get

$$
\begin{aligned}
& \left\langle\hat{q}_{1}^{* m}(t) \hat{q}_{1}^{n}(t)\right\rangle \\
& =e^{i\left(m \hat{z}_{1}^{*}-n \hat{z}_{1}\right) t} \sum_{l=0}^{m i n(m, n)} \frac{m ! n !}{(m-l) !(n-l) ! l !} \\
& \times \hat{q}_{1}^{* m-l}(0) \hat{q}_{1}^{n-l}(0)\left(\frac{k_{B} T}{\hat{\omega}_{1}}\right)^{l}\left(e^{2 \hat{\gamma} t}-1\right)^{l} .
\end{aligned}
$$

Now we can compare the above expression with the time-evolved dressed products $e^{i L_{H} t} \Lambda^{\dagger} q_{1}^{* m} q_{1}^{n}$. We have (see Eq. (68) and Eq. (91))

$$
\begin{aligned}
& e^{i L_{H} t} \Lambda^{\dagger} q_{1}^{* m} q_{1}^{n}=\sum_{a=0}^{n} \frac{m ! n !}{(m-a) !(n-a) ! a !} \\
& \times e^{i\left(m z_{1}^{*}-n z_{1}\right) t} e^{2 \gamma a t} \tilde{Q}^{* m-a} \tilde{Q}^{n-a} Y^{a} .
\end{aligned}
$$

Writing

$$
e^{2 \gamma a t}=\sum_{l=0}^{a} \frac{a !}{l !(a-l) !}\left(e^{2 \gamma t}-1\right)^{l}
$$

and $l^{\prime}=a-l$ we have

$$
\begin{aligned}
& e^{i L_{H} t} \Lambda^{\dagger} q_{1}^{* m} q_{1}^{n}=\sum_{l=0}^{n} \sum_{l^{\prime}=0}^{n-l} \frac{m ! n !}{\left(m-l-l^{\prime}\right) !\left(n-l-l^{\prime}\right) ! l ! l^{\prime} !} \\
& \times e^{i\left(m z_{1}^{*}-n z_{1}\right) t} \tilde{Q}^{* m-l-l^{\prime}} \tilde{Q}^{n-l-l^{\prime}} Y^{l+l^{\prime}}\left(e^{2 \gamma t}-1\right)^{l} \\
& =\sum_{l=0}^{n} \frac{m ! n !}{(m-l) !(n-l) ! l !} e^{i\left(m z_{1}^{*}-n z_{1}\right) t} \\
& \times \sum_{l^{\prime}=0}^{n-l} \frac{(m-l) !(n-l) !}{\left(m-l-l^{\prime}\right) !\left(n-l-l^{\prime}\right) ! l^{\prime} !} \tilde{Q}^{* m-l-l^{\prime}} \tilde{Q}^{n-l-l^{\prime}} Y^{l^{\prime}} \\
& \times Y^{l}\left(e^{2 \gamma t}-1\right)^{l} .
\end{aligned}
$$

Using Eq. (91) again we obtain

$$
\begin{aligned}
& e^{i L_{H} t} \Lambda^{\dagger} q_{1}^{* m} q_{1}^{n} \\
& =e^{i\left(m z_{1}^{*}-n z_{1}\right) t} \sum_{l=0}^{n} \frac{m ! n !}{(m-l) !(n-l) ! l !} \\
& \times\left(\Lambda^{\dagger} q_{1}^{* m-l} q_{1}^{n-l}\right) Y^{l}\left(e^{2 \gamma t}-1\right)^{l} .
\end{aligned}
$$


Comparing Eq. (111) and Eq. (115), we see the direct correspondences

$$
\begin{aligned}
\hat{z}_{1} & \Leftrightarrow z_{1} \\
\frac{k_{B} T}{\hat{\omega}_{1}} & \Leftrightarrow Y=\sum_{k} b_{k} q_{k}^{*} q_{k} \\
\left\langle\hat{q}_{1}^{* m}(t) \hat{q}_{1}^{n}(t)\right\rangle & \Leftrightarrow e^{i L_{H} t} \Lambda^{\dagger}\left(q_{1}^{* m} q_{1}^{n}\right) .
\end{aligned}
$$

The form and time evolution of the ensemble average of Langevin equation variables are the same as those of $\Lambda$ transformed variables. Furthermore, if we take the ensemble average of $\Lambda^{\dagger} q_{1}^{* m} q_{1}^{n}$, we see a closer correspondence. Let us assume that the field action $J_{k}$ obeys the unperturbed Gibbs distribution. The initial distribution $\tilde{\rho}_{0}(\Gamma)$ has the form (with $\beta \equiv 1 /\left(k_{B} T\right)$ )

$$
\tilde{\rho}_{0}(\Gamma)=C \rho_{01}\left(J_{1}, \alpha_{1}\right) \exp \left(-\beta \sum_{k} \omega_{k} J_{k}\right)
$$

where $C$ is a normalization constant, $k_{B}$ is Boltzmann's constant and $T$ is the temperature. As shown in Eq. (E5) the average of $J_{k}$ over this ensemble is

$$
\left\langle J_{k}\right\rangle=\frac{1}{\omega_{k} \beta}=\frac{k_{B} T}{\omega_{k}} .
$$

To calculate $\sum_{k} b_{k}\left\langle J_{k}\right\rangle$, we need the form of $b_{k}$. The approximate value of $b_{k}$ is given in Eq. (90), which for the weak coupling case is approximated by the delta function $(2 \pi / L) \delta\left(\omega_{k}-\tilde{\omega}_{1}\right)$ [15]. So we get

$$
\sum_{k} b_{k}\left\langle J_{k}\right\rangle=\sum_{k} b_{k} \frac{k_{B} T}{\omega_{k}} \approx \frac{k_{B} T}{\tilde{\omega}_{1}} .
$$

Note that $\omega_{k}{ }^{-1}$ does not make any divergence for small $k$ since $b_{k}$ is proportional to $v_{k}^{2} \sim \omega_{k}$ for small $k$.

In short, we obtain a complete correspondence between $\Lambda$ transformed modes and Langevin modes (see Eq. (116)). The systematic removal of Poincaré divergences in the $\Lambda$ transformation gives the Gaussian white noise structure.

\section{THE FOKKER-PLANCK EQUATION}

Using the above results we can now derive the FokkerPlanck equation for the transformed density function $\tilde{\rho}=$ $\Lambda \rho$. We start with the transformed equation (see Eq. (6))

$$
i \frac{\partial}{\partial t} \tilde{\rho}=\tilde{\theta} \tilde{\rho}
$$

We derive the Fokker-Planck equation for $q_{1}, q_{1}^{*}$. We follow the standard derivation found in textbooks (see [26, 37]), but now in terms of $\Lambda$. Consider a test function $G\left(q_{1}, q_{1}^{*}\right)$, which is smooth and vanishes at $\left|q_{1}\right|=\infty$. Multiplying this on both sides of Eq. (120) and integrating over the phase space, we have

$$
\begin{aligned}
& \int d \Gamma G\left(q_{1}, q_{1}^{*}\right) i \frac{\partial}{\partial t} \tilde{\rho}(\Gamma, t)=\int d \Gamma G\left(q_{1}, q_{1}^{*}\right) \tilde{\theta}(\Gamma) \tilde{\rho}(\Gamma, t) \\
& =\int d \Gamma d \Gamma^{\prime} G\left(q_{1}, q_{1}^{*}\right) \tilde{\theta}(\Gamma) \delta\left(\Gamma-\Gamma^{\prime}\right) \tilde{\rho}\left(\Gamma^{\prime}, t\right) .
\end{aligned}
$$

In Eq. (121), $\tilde{\theta}(\Gamma)$ means $\tilde{\theta}$ that acts on $\Gamma$ variables. We expand $G\left(q_{1}, q_{1}^{*}\right)$ near $q_{1}^{\prime}$ and $q_{1}^{\prime *}$.

$$
\begin{aligned}
& \int d \Gamma G\left(q_{1}, q_{1}^{*}\right) i \frac{\partial}{\partial t} \tilde{\rho}(\Gamma, t) \\
& =\int d \Gamma d \Gamma^{\prime}\left\{\sum_{m=0}^{\infty} \sum_{n=0}^{\infty} \frac{1}{m ! n !}\left(\frac{\partial^{m}}{\partial\left(q_{1}^{\prime}\right)^{m}} \frac{\partial^{n}}{\partial\left(q_{1}^{\prime}\right)^{n}} G\left(q_{1}^{\prime}, q_{1}^{\prime}{ }^{*}\right)\right)\right. \\
& \left.\times\left(q_{1}^{*}-q_{1}^{\prime}\right)^{m}\left(q_{1}-q_{1}^{\prime}\right)^{n}\right\} \tilde{\theta}(\Gamma) \delta\left(\Gamma-\Gamma^{\prime}\right) \tilde{\rho}\left(\Gamma^{\prime}, t\right) .
\end{aligned}
$$

Integrating by parts, Eq. (122) becomes

$$
\begin{aligned}
& \int d \Gamma G\left(q_{1}, q_{1}^{*}\right) i \frac{\partial}{\partial t} \tilde{\rho}(\Gamma, t) \\
& =\int d \Gamma^{\prime} G\left(q_{1}^{\prime}, q_{1}^{\prime}{ }^{*}\right) \sum_{m=0}^{\infty} \sum_{n=0}^{\infty} \frac{(-1)^{m+n}}{m ! n !} \frac{\partial^{m}}{\partial\left(q_{1}^{\prime *}\right)^{m}} \frac{\partial^{n}}{\partial\left(q_{1}^{\prime}\right)^{n}} \\
& \times\left[\int d \Gamma\left(q_{1}^{*}-q_{1}^{\prime}{ }^{*}\right)^{m}\left(q_{1}-q_{1}^{\prime}\right)^{n} \tilde{\theta}(\Gamma) \delta\left(\Gamma-\Gamma^{\prime}\right)\right] \tilde{\rho}\left(\Gamma^{\prime}, t\right) .
\end{aligned}
$$

We call the quantities inside the brackets in Eq. (123) the "moments" of order $m+n$. The moments are calculated explicitly in Appendix 【 They are given by

$$
\begin{aligned}
& \int d \Gamma\left(q_{1}^{*}-q_{1}{ }^{*}\right)^{m}\left(q_{1}-q_{1}^{\prime}\right)^{n} \tilde{\theta}(\Gamma) \delta\left(\Gamma-\Gamma^{\prime}\right) \\
& =\left\{\begin{array}{c}
z_{1} q_{1}^{\prime}, \quad m=0, n=1 \\
-z_{1}^{*} q_{1}{ }^{*}, \quad m=1, n=0 \\
2 i \gamma \sum_{k} b_{k} q_{k}{ }^{*} q_{k}^{\prime}, \quad m=1, n=1 \\
0, \quad \text { for all other } m \text { and } n .
\end{array}\right.
\end{aligned}
$$

Substituting Eq. (124) into Eq. (123), we get

$$
\begin{aligned}
& \int d \Gamma G\left(q_{1}, q_{1}^{*}\right) i \frac{\partial}{\partial t} \tilde{\rho}(\Gamma, t) \\
& =\int d \Gamma^{\prime} G\left(q_{1}^{\prime}, q_{1}^{\prime *}\right)\left(-\frac{\partial}{\partial q_{1}^{\prime}}\right)\left(z_{1} q_{1}^{\prime}\right) \tilde{\rho}\left(\Gamma^{\prime}, t\right) \\
& +\int d \Gamma^{\prime} G\left(q_{1}^{\prime}, q_{1}^{\prime *}\right)\left(-\frac{\partial}{\partial q_{1}^{\prime *}}\right)\left(-z_{1}^{*} q_{1}^{\prime}\right) \tilde{\rho}\left(\Gamma^{\prime}, t\right) \\
& +\int d \Gamma^{\prime} G\left(q_{1}^{\prime}, q_{1}^{\prime}\right)\left(\frac{\partial^{2}}{\partial q_{1}^{\prime} \partial q_{1}^{\prime}}\right)\left(2 i \gamma \sum_{k} b_{k} q_{k}^{\prime}{ }^{*} q_{k}^{\prime}\right) \tilde{\rho}\left(\Gamma^{\prime}, t\right) .
\end{aligned}
$$

By changing the integration variable $\Gamma^{\prime}$ to $\Gamma$ in the right hand side of Eq. (125) and eliminating $i$ on both sides, we have

$$
\begin{aligned}
& \int d \Gamma G\left(q_{1}, q_{1}^{*}\right) \frac{\partial}{\partial t} \tilde{\rho}(\Gamma, t) \\
= & \int d \Gamma G\left(q_{1}, q_{1}^{*}\right)\left\{\frac{\partial}{\partial q_{1}}\left(i z_{1} q_{1}\right)+\frac{\partial}{\partial q_{1}^{*}}\left(-i z_{1}^{*} q_{1}^{*}\right)\right. \\
+ & \left.\frac{\partial}{\partial q_{1} \partial q_{1}^{*}}\left(2 \gamma \sum_{k} b_{k} q_{k}^{*} q_{k}\right)\right\} \tilde{\rho}(\Gamma, t) .
\end{aligned}
$$

Now suppose that $\tilde{\rho}(\Gamma, t)$ is factorized at $t=0$. In other words, we write $\tilde{\rho}(\Gamma, 0)$ as

$$
\tilde{\rho}(\Gamma, 0)=g_{1}\left(q_{1}, q_{1}^{*}\right) \prod_{k} g_{k}\left(q_{k}, q_{k}^{*}\right) .
$$




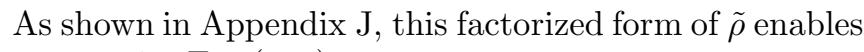
us to write Eq. (126) as

$$
\begin{aligned}
& \int d \Gamma_{1} G\left(q_{1}, q_{1}^{*}\right) \frac{\partial}{\partial t} \int d \Gamma_{f} \tilde{\rho}(\Gamma, t) \\
= & \int d \Gamma_{1} G\left(q_{1}, q_{1}^{*}\right)\left\{\frac{\partial}{\partial q_{1}}\left(i z_{1} q_{1}\right)+\frac{\partial}{\partial q_{1}^{*}}\left(-i z_{1}^{*} q_{1}^{*}\right)\right. \\
+ & \frac{\partial^{2}}{\partial q_{1} \partial q_{1}^{*}}\left(2 \gamma \sum_{k} b_{k}\left\langle q_{k}^{*} q_{k}\right\rangle\right) \int d \Gamma_{f} \tilde{\rho}(\Gamma, t) .
\end{aligned}
$$

Since $G\left(\Gamma_{1}\right)$ is an arbitrary test function, we can write Eq. (128) as

$$
\begin{aligned}
\frac{\partial}{\partial t} \tilde{\rho}_{1}\left(\Gamma_{1}, t\right) & =\left\{i z_{1} \frac{\partial}{\partial q_{1}} q_{1}-i z_{1}^{*} \frac{\partial}{\partial q_{1}^{*}} q_{1}^{*}\right. \\
& \left.+2 \gamma \sum_{k} b_{k}\left\langle J_{k}\right\rangle \frac{\partial}{\partial q_{1} \partial q_{1}^{*}}\right\} \tilde{\rho}_{1}\left(\Gamma_{1}, t\right),
\end{aligned}
$$

where

$$
\tilde{\rho}_{1}\left(\Gamma_{1}, t\right)=\int d \Gamma_{f} \tilde{\rho}(\Gamma, t) .
$$

Eq. (129) is our Fokker-Planck equation for the normal modes. This equation is applicable for any initial field configuration obeying the extensive condition and Eq. (127). In the non-extensive case, the diffusion term containing $b_{k}$ vanishes, and the equation describes damping of the oscillator without Brownian motion. For the special case where the field has the unperturbed Gibbs distribution, using the approximation (119) we get

$$
\begin{aligned}
\frac{\partial}{\partial t} \tilde{\rho}_{1}\left(\Gamma_{1}, t\right) & \approx\left\{i z_{1} \frac{\partial}{\partial q_{1}} q_{1}-i z_{1}^{*} \frac{\partial}{\partial q_{1}^{*}} q_{1}^{*}\right. \\
& \left.+\frac{2 \gamma k_{B} T}{\tilde{\omega}_{1}} \frac{\partial^{2}}{\partial q_{1} \partial q_{1}^{*}}\right\} \tilde{\rho}_{1}\left(\Gamma_{1}, t\right) .
\end{aligned}
$$

This is precisely the equation one obtains from the phenomenological Langevin equation (101).

The Fokker-Planck equation for other variables can be also derived from Eq. (129) by changing variables. For example, the Fokker-Planck equation for the position and momentum $x_{1}$ and $p_{1}$ is given by

$$
\begin{aligned}
\frac{\partial}{\partial t} \tilde{\rho}_{1}\left(\Gamma_{1}, t\right) & =\left\{-\frac{\partial}{\partial x_{1}}\left(\frac{p_{1}}{\tilde{m}}-\gamma x_{1}\right)+\frac{\partial}{\partial p_{1}}\left(\tilde{m} \tilde{\omega}_{1}^{2} x_{1}+\gamma p_{1}\right)\right. \\
& \left.+\frac{D_{x}}{2} \frac{\partial^{2}}{\partial x_{1}^{2}}+\frac{D_{p}}{2} \frac{\partial^{2}}{\partial p_{1}^{2}}\right\} \tilde{\rho}_{1}\left(\Gamma_{1}, t\right),
\end{aligned}
$$

where

$$
\begin{aligned}
\tilde{m} & =m \omega_{1} / \tilde{\omega}_{1} \\
D_{x} & =\frac{2 \gamma}{\tilde{m} \tilde{\omega}_{1}} \sum_{k} b_{k}\left\langle J_{k}\right\rangle \approx \frac{2 \gamma k_{B} T}{\tilde{m} \tilde{\omega}_{1}^{2}}, \\
D_{p} & =2 \tilde{m} \gamma \tilde{\omega}_{1} \sum_{k} b_{k}\left\langle J_{k}\right\rangle \approx 2 \tilde{m} \gamma k_{B} T .
\end{aligned}
$$

[the approximate values are applicable for the unperturbed Gibbs distribution.] The Fokker-Planck equation for the action variable $J_{1}$ is given (after integration over the angle variable $\alpha_{1}$ ) by

$$
\begin{aligned}
& \frac{\partial}{\partial t} \tilde{\rho}\left(J_{1}, t\right) \\
& =\left\{2 \gamma \frac{\partial}{\partial J_{1}}\left(J_{1}-\frac{k_{B} T}{\tilde{\omega}_{1}}\right)+D_{J} \frac{\partial^{2}}{\partial J_{1}^{2}} J_{1}\right\} \tilde{\rho}\left(J_{1}, t\right),
\end{aligned}
$$

where

$$
D_{J}=2 \gamma \sum_{k} b_{k}\left\langle J_{k}\right\rangle \approx \frac{2 \gamma k_{B} T}{\tilde{\omega}_{1}} .
$$

Eqs. (132) and (134) coincide (in the weak-coupling approximation) with the equations for Brownian motion of an oscillator in an anharmonic lattice derived in Ref. 35]. Eq. (134) (in its exact form) was first proposed by T. Petrosky [33].

Note that Eq. 1321 is symmetric with respect to rescaled position $x_{1}$ and momentum $p_{1}$. The reason is that the Hamiltonian considered here is symmetric in rescaled $x_{1}$ and $p_{1}$ to begin with. The same is true for the anharmonic lattice model considered in Ref. 35|. In contrast, the Kramers (Fokker-Planck) equation 35, 38. derived from the Ornstein-Uhlenbeck phenomenological theory of Brownian motion [39] is not symmetric, because the Brownian force breaks the position-momentum symmetry. In spite of the difference, for the case $\gamma \ll \omega_{1}$, Eq. (132) gives the same solution as the Kramers equation. The solutions of Eq. (132) can be found in Ref. 35.

\section{CONCLUSIONS}

In this paper we studied the irreversible and stochastic behavior of an oscillator coupled to a field in the thermodynamic limit, using the star-unitary transformation $\Lambda$. We showed that the average of dressed particle modes has the same time evolution as the ensemble average of particle modes in the Langevin equation (101). Also, the reduced distribution function for the particle variables exactly obeys the Fokker-Planck equation (129), which describes the damping and diffusion processes. It is remarkable that the systematic removal of Poincaré divergences by analytic continuation leads to the same structure as that of Gaussian white noise.

Since the Gaussian structure of the fluctuations is coming from the resonances, rather than the specific form of the initial field ensemble, our derivation of the FokkerPlanck equation is valid for both Gaussian and nonGaussian field ensembles. Due to the Fokker-Planck evolution, the particle distribution is still Gaussian for $t \rightarrow \infty$.

Our method of isolating Poincaré divergences can in principle be applied to more complicated systems than the one considered here, in order to investigate further the relation between noise and dynamics. 
We have studied the fluctuations of a particle surrounded by a field. One can also consider the fluctuations of the field modes induced by the particle. An interesting result is that fluctuations of the $\Lambda$-transformed field modes appear even when the energy of the field is nonextensive. If we go back to the initial formulation, acting with $\Lambda^{-1}$, we recover the usual equations. This will be discussed elsewhere 22].

Markovian equations such as the Langevin or FokkerPlanck equations describe irreversible and stochastic processes. Rather than viewing these equations as approximations of Hamiltonian dynamics, we see them as describing components of dynamics. These components can be identified through a change of phase-space variables obtained by the $\Lambda$ transformation. This gives a representation with broken time-symmetry.

In the non-Markovian equations, the effects of dressing are not separated from irreversible (or thermodynamic) processes. In our approach the dressing on the particle is incorporated from the beginning, since we deal with renormalized particles. This allows us to isolate pure thermodynamic behavior such as Brownian motion with a white noise source. In this sense, we can aim to reformulate thermodynamics in terms of renormalized particles. This is interesting in view of recent claims 7] that traditional thermodynamics may not apply for quantum Brownian motion at low temperatures, due to the nonMarkovian character of quantum noise.

The relation with thermodynamics requires a precise definition of entropy or $\mathcal{H}$ function. The latter can be given in terms of $\Lambda$ [16]. The introduction of $\Lambda$ leads to probabilistic considerations in classical mechanics, independent of incomplete knowledge or quantum corrections. In conclusion, we believe that our approach leads to a unification of dynamics, thermodynamics and noise.

\section{Acknowledgments}

We thank Prof. I. Prigogine and Dr. T. Petrosky for their support and encouragement, as well as for many helpful comments and suggestions. We thank the referee that reviewed our paper, for many comments that have helped us to clarify our ideas and improve our presentation. We thank also Dr. G. Akguc, Prof. I. Antoniou, Prof. R. Balescu, Dr. D. Driebe, Prof. M. de Haan, Dr. E. Karpov, Mr. C. B. Li, Prof. G. Nicolis, Prof. E.C.G. Sudarshan, Prof. S. Tasaki, and Mr. C. Ting for helpful comments. We acknowledge the International Solvay Institutes for Physics and Chemistry, the Engineering Research Program of the Office of Basic Energy Sciences at the U.S. Department of Energy, Grant No DE-FG03-94ER14465, the Robert A. Welch Foundation Grant F-0365, The European Commission Project HPHA-CT-2001-40002, the National Lottery of Belgium and the Communauté Francaise de Belgique for supporting this work.

\section{APPENDIX A: EXACT TIME EVOLUTION OF PARTICLE AND FIELD MODES}

We consider first the integrable case. The time evolution of the modes $q_{s}(t)$ can be calculated using the renormalized modes $\bar{Q}_{s}$, which are eigenstates of the Liouville operator. We have

$$
e^{i L_{H} t} \bar{Q}_{s}=e^{-i \bar{\omega}_{s} t} \bar{Q}_{s}
$$

The particle mode $\bar{Q}_{1}$ is given Eq. (47). The field modes $\bar{Q}_{k}$ are found from the equation $L_{H} \bar{Q}_{k}=-\bar{\omega}_{k} \bar{Q}_{k}$, which gives

$$
\bar{Q}_{k}=N_{k}^{1 / 2}\left[q_{k}+\frac{\lambda V_{k}}{\eta_{k}\left(\bar{\omega}_{k}\right)}\left(q_{1}+\sum_{k^{\prime}(\neq k)} \frac{\lambda V_{k^{\prime}}}{\bar{\omega}_{k}-\omega_{k^{\prime}}} q_{k^{\prime}}\right)\right]
$$

where

$$
\begin{gathered}
\eta_{k}(z) \equiv z-\omega_{1}-\sum_{k^{\prime}(\neq k)} \frac{\lambda^{2} V_{k^{\prime}}^{2}}{z-\omega_{k^{\prime}}} \\
\bar{\omega}_{k}=\omega_{k}+\frac{\lambda^{2} V_{k}^{2}}{\eta_{k}\left(\bar{\omega}_{k}\right)} \\
N_{k}=\left[1+\frac{\lambda^{2} V_{k}^{2}}{\eta_{k}^{2}\left(\bar{\omega}_{k}\right)}\left(1+\sum_{k^{\prime}(\neq k)} \frac{\lambda^{2} V_{k^{\prime}}^{2}}{\left(\bar{\omega}_{k}-\omega_{k^{\prime}}\right)^{2}}\right)\right]^{-1}
\end{gathered}
$$

Note that $\tilde{\omega}_{k}=\omega_{k}+O(1 / L)$ and also $N_{k}=1+O(1 / L)$. As long as $L$ is finite all the denominators are nonvanishing, and there are no resonances.

We write the linear relations between dressed and bare modes as

$$
\bar{Q}_{s}=\sum_{r} c_{s r} q_{r}, \quad q_{s}=\sum_{r} c_{r s}^{*} \bar{Q}_{r} .
$$

Then, using Eq. A1 we get the coefficients $f_{s r}(t)$ in the equation $q_{s}(t)=\sum_{r} f_{s r}(t) q_{r}(0)$ as

$$
f_{s r}(t)=\sum_{r^{\prime}} c_{r r^{\prime}} e^{-i \bar{\omega}_{r^{\prime}} t} c_{r^{\prime} s}^{*}
$$

In the nonintegrable case resonances appear (see Sec. V] and if we insist on keeping the renormalized modes as usual canonical variables, then the particle modes dissappear into the continuum of field modes (we can however introduce the Gamow modes of Sec. [V] which are noncanonical). Keeping canonical modes, the Hamiltonian is represented as 2, 15, 22]

$$
H=\sum_{k} \omega_{k} \tilde{Q}_{k}^{*} \tilde{Q}_{k}
$$

in the continuous limit. The renormalized field modes are given by

$$
\tilde{Q}_{k}=q_{k}+\frac{\lambda V_{k}}{\eta^{\mp}\left(\omega_{k}\right)}\left[q_{1}+\sum_{k^{\prime}} \frac{\lambda V_{k^{\prime}}}{\omega_{k}-\omega_{k^{\prime}} \mp i \epsilon} q_{k^{\prime}}\right]
$$


where $\epsilon$ is a positive infinitesimal quantity. There are two branches, corresponding to analytical continuation to the lower or upper half planes of $\omega_{k}$. For $t>0$, and given the initial condition Eq. (41), it is convenient to take the branch with $-i \epsilon$, since this will give exponential decay of the particle modes the positive $t$ direction. We get

$$
\begin{aligned}
& q_{1}(t)=\sum_{k} \frac{\lambda V_{k}}{\eta^{+}\left(\omega_{k}\right)} \tilde{Q}_{k}(t)=\sum_{k} \frac{\lambda V_{k}}{\eta^{+}\left(\omega_{k}\right)} \tilde{Q}_{k}(0) e^{-i \omega_{k} t} \\
& =\sum_{k} \frac{\lambda V_{k}}{\eta^{+}\left(\omega_{k}\right)} q_{k}(0) e^{-i \omega_{k} t}+\sum_{k} \frac{\lambda^{2} V_{k}^{2}}{\left|\eta^{+}\left(\omega_{k}\right)\right|^{2}} q_{1}(0) e^{-i \omega_{k} t} \\
& +\sum_{k} \frac{\lambda^{2} V_{k}^{2}}{\left|\eta^{+}\left(\omega_{k}\right)\right|^{2}} \sum_{k^{\prime}} \frac{\lambda V_{k^{\prime}}}{\omega_{k}-\omega_{k^{\prime}}-i \epsilon} q_{k^{\prime}}(0) e^{-i \omega_{k} t}, \quad \text { (A10) }
\end{aligned}
$$

For $t<0$, we take the branch with $+i \epsilon$.

\section{APPENDIX B: RANDOMNESS IN THE FIELD MODES}

We choose our initial condition with the form (41). In classical mechanics $q_{10} \equiv q_{1}(0)$ can be determined exactly since $q_{10}$ is a function of the initial position and momentum of the particle. For the modes $q_{k 0}$ we need more care. Let us first write $q_{k 0}$ in terms of action and angle variables,

$$
q_{k 0}=\sqrt{J_{k 0}} e^{-i \alpha_{k 0}} .
$$

With the ensemble (41) we have

$$
\lim _{L \rightarrow \infty}\left\langle J_{k 0}\right\rangle \sim O\left(L^{0}\right)
$$

in the thermodynamic limit. For example, for an unperturbed Gibbs thermal distribution of the field modes we have $\left\langle J_{k 0}\right\rangle=k_{B} T / \omega_{k}$ (see Eq. (E5)).

For almost all phase points $\left\{J_{10}, . . J_{k 0} . ., \alpha_{10}, . ., \alpha_{k 0}, ..\right\}$ out of the ensemble, any two different angles $\alpha_{k 0}$ and $\alpha_{k^{\prime} 0}$ have no correlation. In other words, the sequence of angles $\left\{\alpha_{k_{n} 0}\right\}$ is completely random for almost all cases. This property allows the existence of the thermodynamic limit [18]. Indeed, if $\alpha_{k 0}$ is uniformly distributed over $[-\pi, \pi]$ and the sequence of angles $\left\{\alpha_{k_{n} 0}\right\}$ is completely random, then the term

$$
\begin{aligned}
& \sum_{k} \frac{\lambda V_{k}}{\eta^{+}\left(\omega_{k}\right)} q_{k 0} e^{-i \omega_{k} t} \\
& =\sum_{k} \frac{\lambda V_{k}}{\eta^{+}\left(\omega_{k}\right)} \sqrt{J_{k 0}} e^{-i\left(\omega_{k}+\alpha_{k 0}\right) t}
\end{aligned}
$$

in Eq. A10 is $O\left(L^{0}\right)$. This is so because the summation is taken over a sequence complex numbers with random phases and hence it is proportional to the square root of the number of modes, which in turn is proportional to $L$. Since $V_{k} \sim L^{-1 / 2}$, Eq. (B33) is $O\left(L^{0}\right)$. It shows a very irregular time evolution as the number of modes increases.
Note that if $\alpha_{k 0}$ was a smooth function of $k$, then for the first term of Eq. A10 we would have in the limit $L \rightarrow \infty$

$$
\begin{aligned}
& \sum_{k} \frac{\lambda V_{k}}{\eta^{+}\left(\omega_{k}\right)} q_{k 0} \\
& =\sqrt{\frac{L}{2 \pi}} \frac{2 \pi}{L} \sum_{k} \frac{\lambda v_{k}}{\eta^{+}\left(\omega_{k}\right)} \sqrt{J_{k 0}} e^{-i \alpha_{k 0}} \\
& \rightarrow \sqrt{\frac{L}{2 \pi}} \int d k \frac{\lambda v_{k}}{\eta^{+}\left(\omega_{k}\right)} \sqrt{J_{k 0}} e^{-i \alpha_{k 0}}
\end{aligned}
$$

and since the integral is $O(1)$, this expression would diverge as $O(\sqrt{L})$.

\section{APPENDIX C: DERIVATION OF THE NON-MARKOVIAN FOKKER-PLANCK EQUATION}

From the Liouville equation we have

$$
\begin{aligned}
& \int d \Gamma G\left(\Gamma_{1}\right) \frac{\partial}{\partial t} \rho(\Gamma, t)=-i \int d \Gamma G\left(\Gamma_{1}\right) L_{H} \rho(\Gamma, t) \\
& =\sum_{m=0}^{\infty} \sum_{n=0}^{\infty} G_{m n} M_{m n}(t)
\end{aligned}
$$

where

$$
M_{m n}(t) \equiv-i \int d \Gamma q_{1}^{* m} q_{1}^{n} L_{H} \rho(\Gamma, t) .
$$

Using Eq. (30) we have

$$
M_{m n}(t)=\int d \Gamma\left(i L_{H}\left[q_{1}^{*}(t)\right]^{m}\left[q_{1}(t)\right]^{n}\right) \rho(\Gamma, 0) .
$$

Since $L_{H}$ is a differential operator, we have

$$
\begin{aligned}
i L_{H}\left[q_{1}^{*}(t)\right]^{m}\left[q_{1}(t)\right]^{n} & =m\left[q_{1}^{*}(t)\right]^{m-1}\left[q_{1}(t)\right]^{n} i L_{H} q_{1}^{*}(t) \\
& +n\left[q_{1}^{*}(t)\right]^{m}\left[q_{1}(t)\right]^{n-1} i L_{H} q_{1}(t) .
\end{aligned}
$$

From Eqs. (31) and (34) we have

$$
\begin{aligned}
& i L_{H} q_{1}^{*}(t)=i z_{1}^{*}(t) q_{1}^{*}(t)+R^{*}(t), \\
& i L_{H} q_{1}(t)=-i z_{1}(t) q_{1}(t)+R(t) .
\end{aligned}
$$

Inserting Eqs. (C4) and (C5) in Eq. (C3) we get

$$
M_{m n}(t)=M_{m n}^{z}(t)+M_{m n}^{R}(t)
$$

where

$$
\begin{aligned}
& M_{m n}^{z}(t) \\
& =\int d \Gamma\left[i z_{1}^{*}(t) m-i z_{1}(t) n\right]\left[q_{1}^{*}(t)\right]^{m}\left[q_{1}(t)\right]^{n} \rho(\Gamma, 0) \\
& =\int d \Gamma\left[i z_{1}^{*}(t) m-i z_{1}(t) n\right] q_{1}^{* m} q_{1}^{n} \rho(\Gamma, t)
\end{aligned}
$$


and

$$
\begin{aligned}
& M_{m n}^{R}(t)=M_{m n}^{R, 1}(t)+M_{m n}^{R, 2}(t), \\
& M_{m n}^{R, 1}(t)=\int d \Gamma m\left[q_{1}^{*}(t)\right]^{m-1}\left[q_{1}(t)\right]^{n} R^{*}(t) \rho(\Gamma, 0), \\
& M_{m n}^{R, 2}(t)=\int d \Gamma n\left[q_{1}^{*}(t)\right]^{m}\left[q_{1}(t)\right]^{n-1} R(t) \rho(\Gamma, 0) .
\end{aligned}
$$

Now we evaluate $M_{m n}^{R, 1}(t)$ in Eq. (C8). Using Eq. (36) we have

$$
\begin{aligned}
& M_{m n}^{R, 1}(t) \\
& =\int d \Gamma m\left[q_{1}^{*}(t)\right]^{m-1}\left[q_{1}(t)\right]^{n} \sum_{k} h_{k}^{*}(t) q_{k}^{*} \rho(\Gamma, 0) .
\end{aligned}
$$

Then, using Eq. (32) we obtain

$$
\begin{aligned}
M_{m n}^{R, 1}(t) & =\int d \Gamma m\left[f_{11}^{*}(t) q_{1}^{*}+\sum_{p} f_{1 p}^{*}(t) q_{p}^{*}\right]^{m-1} \\
& \times\left[f_{11}(t) q_{1}+\sum_{p^{\prime}} f_{1 p^{\prime}}(t) q_{p^{\prime}}\right]^{n} \\
& \times \sum_{k} h_{k}^{*}(t) q_{k}^{*} \rho(\Gamma, 0)
\end{aligned}
$$

Abbreviating $f_{\alpha} \equiv f_{1 \alpha}(t), h_{k}(t) \equiv h_{k}$ and using binomial expansions, we get

$$
\begin{aligned}
& M_{m n}^{R, 1}(t)=\int d \Gamma \sum_{a=0}^{m-1} \sum_{b=0}^{n} \frac{(m-1) !}{(m-1-a) ! a !} \frac{n !}{(n-b) ! b !} \\
& \times m\left[f_{1}^{*} q_{1}^{*}\right]^{m-1-a}\left[f_{1} q_{1}\right]^{n-b} \\
& \times \sum_{p_{1} \cdots p_{a+1}} \sum_{p_{1}^{\prime} \cdots p_{b}^{\prime}} f_{p_{1}}^{*} \cdots f_{p_{a}}^{*} h_{p_{a+1}}^{*} f_{p_{1}^{\prime}} \cdots f_{p_{b}^{\prime}} \\
& \times q_{p_{1}}^{*} \cdots q_{p_{a}}^{*} q_{p_{a+1}}^{*} q_{p_{1}^{\prime}} \cdots q_{p_{b}^{\prime}} \rho(\Gamma, 0)
\end{aligned}
$$

where we changed the variable $k$ to $p_{a+1}$ Due to the assumed form of the field distribution in Eq. (41), only expectation values of observables independent of the angles $\alpha_{p}$ of the field modes are nonzero (in other words, each $q_{p_{j}^{\prime}}$ field mode must be multiplied by its complex conjugate $q_{p_{i}}^{*}$ with $p_{j}^{\prime}=p_{i}$ ). Thus in Eq. (C11) we must have $b=a+1$ and we get $(a+1)$ ! possible parings

$$
\begin{aligned}
& M_{m n}^{R, 1}(t)=\int d \Gamma \sum_{a=0}^{\min (m-1, n-1)} \\
& \times \frac{(m-1) !}{(m-1-a) ! a !} \frac{n !}{(n-1-a) !(a+1) !} \\
& \times m\left[f_{1}^{*} q_{1}^{*}\right]^{m-1-a}\left[f_{1} q_{1}\right]^{n-1-a} \\
& \times \sum_{p_{1} \cdots p_{a+1}}\left|f_{p_{1}}\right|^{2} \cdots\left|f_{p_{a}}\right|^{2} h_{p_{a+1}}^{*} f_{p_{a+1}} \\
& \times(a+1) ! q_{p_{1}}^{*} q_{p_{1}} \cdots q_{p_{a}}^{*} q_{p_{a}} q_{p_{a+1}}^{*} q_{p_{a+1}}^{*} \rho(\Gamma, 0) .
\end{aligned}
$$

Note that due to the volume dependence of the coefficients $f_{p} \sim O(1 / \sqrt{L})$ and $h_{p} \sim 1 / \sqrt{L}$ [which follows from Eq. (A10)], we can neglect the cases where two or more of the wave numbers are repeated, e.g., $p_{i}=p_{j}$ for $i \neq j$. Indeed, these cases give contributions of order $O(1 / L)$ within the summations in Eq. (C12). Canceling $(a+1)$ ! and writing $k=p_{a+1}$ and $n !=n(n-1)$ we get

$$
\begin{aligned}
& M_{m n}^{R, 1}(t)=m n \int d \Gamma \sum_{a=0}^{m i n(m-1, n-1)} \\
& \times \frac{(m-1) !}{(m-1-a) ! a !} \frac{(n-1) !}{(n-1-a) !} \\
& \times\left[f_{1}^{*} q_{1}^{*}\right]^{m-1-a}\left[f_{1} q_{1}\right]^{n-1-a} \\
& \times \sum_{p_{1} \cdots p_{a}}\left|f_{p_{1}}\right|^{2} \cdots\left|f_{p_{a}}\right|^{2} \\
& \times q_{p_{1}}^{*} q_{p_{1}} \cdots q_{p_{a}}^{*} q_{p_{a}} \sum_{k} h_{k}^{*} f_{k} q_{k}^{*} q_{k} \rho(\Gamma, 0) .
\end{aligned}
$$

Applying the reverse steps from Eqs. (C12 to (C10) we get

$$
\begin{aligned}
M_{m n}^{R, 1}(t) & =m n \int d \Gamma\left[q_{1}^{*}(t)\right]^{m-1}\left[q_{1}(t)\right]^{n-1} \\
& \times \sum_{k} h_{k}^{*}(t) f_{1 k}(t) q_{k}^{*} q_{k} \rho(\Gamma, 0) \\
& =m n \int d \Gamma\left[q_{1}^{*}(t)\right]^{m-1}\left[q_{1}(t)\right]^{n-1} \\
& \times \sum_{k} h_{k}^{*}(t) f_{1 k}(t)\left\langle q_{k}^{*} q_{k}\right\rangle \rho(\Gamma, 0)+O(1 / L)
\end{aligned}
$$

where due to the factorization property Eq. (41) we can take independently the average

$$
\left\langle q_{k}^{*} q_{k}\right\rangle=\left\langle J_{k}\right\rangle=\int d \Gamma q_{k}^{*} q_{k} \rho(\Gamma, 0) .
$$

[a similar argument is given in Appendix [J. Bringing the time dependence back to $\rho$ we get then

$$
\begin{aligned}
M_{m n}^{R, 1}(t) & =m n \int d \Gamma\left[q_{1}^{*}\right]^{m-1}\left[q_{1}\right]^{n-1} \\
& \times \sum_{k} h_{k}^{*}(t) f_{1 k}(t)\left\langle J_{k}\right\rangle \rho(\Gamma, t) .
\end{aligned}
$$

For the second term in Eq. (C8) we have $M_{m n}^{R, 2}(t)=$ $\left[M_{n m}^{R, 1}(t)\right]^{*}$. Putting everything together in Eq. (C1) with integration by parts and a few straightforward manipulations, we obtain

$$
\begin{aligned}
& \int d \Gamma G\left(\Gamma_{1}\right) \frac{\partial}{\partial t} \rho(\Gamma, t) \\
= & \int d \Gamma G\left(\Gamma_{1}\right)\left\{i z_{1}(t) \frac{\partial}{\partial q_{1}} q_{1}-i z_{1}^{*}(t) \frac{\partial}{\partial q_{1}^{*}} q_{1}^{*}\right. \\
+ & \left.D(t) \frac{\partial^{2}}{\partial q_{1} \partial q_{1}^{*}}\right\} \rho(\Gamma, t) .
\end{aligned}
$$

where

$$
D(t)=\sum_{k}\left[h_{k}^{*}(t) f_{1 k}(t)+\text { c.c. }\right]\left\langle J_{k}\right\rangle \text {. }
$$


Since $G\left(\Gamma_{1}\right)$ is an arbitrary test function, we come to the non-Markovian equation (42) for the reduced distribution function $\rho_{1}$. Replacing the explicit form of $h_{k}^{*}(t)$ we obtain as well Eq. (43).

\section{APPENDIX D: MARKOVIAN APPROXIMATION}

Phenomenological equations may be obtained from dynamics using the so-called Markovian approximation (or the $\lambda^{2} t$ approximation studied by Van Hove and others 37, 40, 41, 42 ), where memory effects are neglected (see also [43, 44, 45]). This approximation is valid if we take the continuous spectrum limit $L \rightarrow \infty$ (with Poincaré resonances), for weak coupling and for time scales where the relaxation process dominates over dressing processes (the particle-bath correlations are negligible [4, 35]).

In this appendix we consider the Markovian approximation of the dynamical evolution of the bare particle modes. We will write the weak coupling approximation of the coefficients $f_{11}(t)$ and $f_{1 k}(t)$, which will give the Markovian approximation of Eqs. (34) and (42).

We start with Eq. A10, for $t>0$. We approximate for weak coupling $\lambda \ll 1$,

$$
\frac{1}{\eta^{+}\left(\omega_{k}\right)} \approx \frac{1}{\omega_{k}-z_{1}}
$$

We then separate the pole contribution at $\omega_{k}=z_{1}$ and the branch cut contribution from each term. The pole contribution gives the exponential decaying part and the cut contribution gives classical Zeno effect and nonexponential behavior [22]. Here we will only consider the pole contributions in Eq. (A10), which amounts to the Markovian approximation. Taking the pole contribution at $\omega_{k}=z_{1}$ in the last two terms of Eq. A10, we get

$$
\begin{aligned}
& q_{1}(t) \approx q_{1}(0) e^{-i z_{1} t}+\sum_{k} \frac{\lambda V_{k}}{\omega_{k}-z_{1}} q_{k}(0) e^{-i \omega_{k} t} \\
& -\sum_{k} \frac{\lambda V_{k}}{\omega_{k}-z_{1}} q_{k}(0) e^{-i z_{1} t} .
\end{aligned}
$$

Therefore we have

$$
\begin{aligned}
& f_{11}(t) \approx e^{-i z_{1} t}, \\
& f_{1 k}(t) \approx \sum_{k} \frac{\lambda V_{k}}{\omega_{k}-z_{1}}\left[e^{-i \omega_{k} t}-e^{-i z_{1} t}\right] .
\end{aligned}
$$

This leads to

$$
\begin{aligned}
& z_{1}(t) \approx z_{1}, \\
& R(t) \approx-i \sum_{k} \lambda V_{k} e^{-i \omega_{k} t} q_{k}(0) .
\end{aligned}
$$

For $t<0$ we can repeat the same procedure, except that now we choose the $+i \epsilon$ branch in Eq. A9). Then we obtain the following approximate equation

$$
\begin{array}{ll}
\dot{q}_{1}(t) \approx-i z_{1} q_{1}(t)+R(t), & t>0 \\
\dot{q}_{1}(t) \approx-i z_{1}^{*} q_{1}(t)+R(t), & t<0
\end{array}
$$

which has a Langevin form. This equation is expressed in two branches, one for $t>0$ and the other for $t<0$. The change $-i z_{1} \Rightarrow-i z_{1}^{*}$ corresponds to the change $\gamma \Rightarrow-\gamma$, since $z_{1}=\tilde{\omega}_{1}-i \gamma$. Taken separately, each branch breaks time reversal invariance, while as a whole time reversal invariance is kept [note that in the integrable case we have $\gamma=0$ and there is no splitting into two branches]. Comparing Eq. (D5) with Eq. (101) we identify $\hat{z}_{1}=z_{1}$. Furthermore, the term $R(t)$ behaves as the white-noise source $\hat{R}(t)$ in the sense that in the pole approximation, the auto-correlation functions of the variables $\hat{q}_{1}$ and $q_{1}$ coincide. Indeed, the "noise" term $R(t)$ in Eq. (D4) has the same Gaussian property (103) as the noise $\hat{R}(t)$, provided we replace noise averages with averages with ensembles of the form (41) (see discussion below Eq. (C11)). Then, defining

$$
\begin{aligned}
& q_{1 a}(t) \equiv q_{1}(0) e^{-i z_{1} t} \\
& q_{1 r}(t) \equiv e^{-i z_{1} t} \int_{0}^{t} d t^{\prime} R\left(t^{\prime}\right) e^{i z_{1} t^{\prime}} \\
& =\sum_{k} \frac{\lambda V_{k}}{\omega_{k}-z_{1}}\left[e^{-i \omega_{k} t}-e^{-i z_{1} t}\right] q_{k}(0)
\end{aligned}
$$

and following the same steps as in Eqs. (106)- (110) we get

$$
\left\langle q_{1 r}^{* k}(t) q_{1 r}^{l}(t)\right\rangle=l ! \delta_{k l}\left\langle q_{1 r}^{*}(t) q_{1 r}(t)\right\rangle^{l} .
$$

In general we have (with $q_{k 0} \equiv q_{k}(0)$ )

$$
\begin{aligned}
& \left\langle q_{1 r}^{*}(t+\tau) q_{1 r}(t)\right\rangle \\
& \approx\left\langle\sum_{k} \frac{\lambda V_{k}}{\omega_{k}-z_{1}^{*}} q_{k 0}^{*}\left(e^{i \omega_{k}(t+\tau)}-e^{i z_{1}^{*}(t+\tau)}\right)\right. \\
& \left.\times \sum_{l} \frac{\lambda V_{l}}{\omega_{l}-z_{1}} q_{l 0}\left(e^{-i \omega_{l} t}-e^{-i z_{1} t}\right)\right\rangle .
\end{aligned}
$$

For the normalized thermal field ensemble we have

$$
\left\langle q_{k 0}^{*} q_{k^{\prime} 0}\right\rangle=\delta_{k k^{\prime}} \frac{k_{B} T}{\omega_{k}} .
$$

Using this result and going to the continuous limit we obtain from Eq. (D9)

$$
\begin{aligned}
& \left\langle q_{1 r}^{*}(t+\tau) q_{1 r}(t)\right\rangle \\
& \approx \int_{0}^{\infty} d w \frac{\lambda^{2} v_{w}^{2}}{\left|\omega-z_{1}\right|^{2}} \frac{k_{B} T}{\omega} \\
& \times\left(e^{i \omega \tau}-e^{-i z_{1} t} e^{i \omega(t+\tau)}-e^{i z_{1}^{*}(t+\tau)} e^{-i \omega t}+e^{i z_{1}^{*} \tau} e^{-2 \gamma t}\right) .
\end{aligned}
$$

For $\gamma \ll \tilde{\omega}_{1}$ the integrand is sharply peaked around $\omega=$ $\tilde{\omega}_{1}$. We separate the pole and the cut contributions to the integral, rewriting Eq. (D11) as

$$
\begin{aligned}
& \left\langle q_{1 r}^{*}(t+\tau) q_{1 r}(t)\right\rangle \\
& \approx\left\langle q_{1 r}^{*}(t+\tau) q_{1 r}(t)\right\rangle_{\text {pole }}+\left\langle q_{1 r}^{*}(t+\tau) q_{1 r}(t)\right\rangle_{c u t}
\end{aligned}
$$


where

$$
\begin{aligned}
& \left\langle q_{1 r}^{*}(t+\tau) q_{1 r}(t)\right\rangle_{\text {pole }} \\
& =\int_{-\infty}^{\infty} d \omega \frac{\lambda^{2} v_{\omega}^{2}}{\left|\omega-z_{1}\right|^{2}} \frac{k_{B} T}{\omega} \\
& \times\left(e^{i \omega \tau}-e^{-i z_{1} t} e^{i \omega(t+\tau)}-e^{i z_{1}^{*}(t+\tau)} e^{-i \omega t}+e^{i z_{1}^{*} \tau} e^{-2 \gamma t}\right),
\end{aligned}
$$

$$
\begin{aligned}
& \left\langle q_{1 r}^{*}(t+\tau) q_{1 r}(t)\right\rangle_{c u t} \\
& =-\int_{-\infty}^{0} d \omega \frac{\lambda^{2} v_{\omega}^{2}}{\left|\omega-z_{1}\right|^{2}} \frac{k_{B} T}{\omega} \\
& \times\left(e^{i \omega \tau}-e^{-i z_{1} t} e^{i \omega(t+\tau)}-e^{i z_{1}^{*}(t+\tau)} e^{-i \omega t}+e^{i z_{1}^{*} \tau} e^{-2 \gamma t}\right) .
\end{aligned}
$$

Using

$$
\begin{aligned}
v_{z_{1}} & \approx v_{\tilde{\omega}_{1}}, \quad \frac{k_{B} T}{z_{1}} \approx \frac{k_{B} T}{\tilde{\omega}_{1}}, \\
\gamma & \approx \pi \lambda^{2} v_{\tilde{\omega}_{1}}^{2},
\end{aligned}
$$

the pole contributions at $\omega=z_{1}, \omega=z_{1}^{*}$ give

$$
\left\langle q_{1 r}^{*}(t+\tau) q_{1 r}(t)\right\rangle_{p o l e} \approx \frac{k_{B} T}{\tilde{\omega}_{1}} e^{i z_{1}^{*} \tau}\left(1-e^{-2 \gamma t}\right) .
$$

The cut part involves the tail of the Lorentzian distribution $1 /\left|\omega-z_{1}\right|^{2}=1 /\left(\left(\omega-\tilde{\omega}_{1}\right)^{2}+\gamma^{2}\right)$. It gives

$$
\begin{aligned}
& \left\langle q_{1 r}^{*}(t+\tau) q_{1 r}(t)\right\rangle_{c u t} \\
& \approx \int_{0}^{\infty} d \omega \frac{\lambda^{2} v_{-\omega}^{2}}{\left|\omega+z_{1}\right|^{2}} \frac{k_{B} T}{\omega} \\
& \times\left(e^{-i \omega \tau}-e^{-i z_{1} t} e^{-i \omega(t+\tau)}-e^{i z_{1}^{*}(t+\tau)} e^{i \omega t}+e^{i z_{1}^{*} \tau} e^{-2 \gamma t}\right) .
\end{aligned}
$$

In weak coupling case $\left(\gamma \ll \tilde{\omega}_{1}\right)$, the cut contribution is much smaller than the pole contribution. Replacing the result (D16) in Eq. (D8) with $\tau=0$ we obtain the same correlation Eq. (111) obtained from the phenomenological Langevin equation.

We have also

$$
D(t) \approx \frac{2 \gamma k_{B} T}{\tilde{\omega}_{1}}
$$

Hence we recover the Markovian Fokker-Planck equation (131) with $\hat{z}_{1}=z_{1}$.

\section{APPENDIX E: PROOF OF EQ. (81)}

In this appendix we show, by perturbation expansion that Eq. 81] is satisfied for the equilibrium Gibbs ensemble. The same relation is valid for the class of ensembles (generally non-equilibrium ensembles) having deltasingularities in the wave numbers, of which the Gibbs ensemble is a special case [13, 14, 35]. The Gibbs ensemble is given by

$$
\rho^{\mathrm{eq}}=C \exp (-\beta H)
$$

where $C$ is a normalization constant, such that

$$
\int d \Gamma \rho^{\mathrm{eq}}(\Gamma)=1
$$

In the perturbation expansion we have

$$
\rho^{\mathrm{eq}}=C \exp \left(-\beta H_{0}\right)\left(1+\lambda V+\frac{1}{2 !} \lambda^{2} V^{2}+\cdots\right) .
$$

Due to the angle integrations, only diagonal monomials are nonzero

$$
\int d \Gamma \prod_{r} q_{r}^{* m_{r}} q_{r}^{n_{r}} \exp \left(-\beta H_{0}\right) \propto \prod_{r} \delta_{m_{r}, n_{r}} .
$$

Considering the explicit form of the potential $V$ we then have

$$
\begin{gathered}
\left\langle J_{k}\right\rangle=\int d \Gamma J_{k} \rho^{\mathrm{eq}} \\
=\frac{\int d \Gamma J_{k} \exp \left(-\beta H_{0}\right)}{\int d \Gamma \exp \left(-\beta H_{0}\right)}+O\left(\lambda^{2}\right) \\
=\frac{\int_{0}^{\infty} d J_{k} J_{k} \exp \left(-\beta \omega_{k} J_{k}\right)}{\int_{0}^{\infty} d J_{k} \exp \left(-\beta \omega_{k} J_{k}\right)}+O\left(\lambda^{2}\right) \\
=\frac{1}{\beta \omega_{k}}+O\left(\lambda^{2}\right) \\
\left\langle q_{k}^{*} q_{k^{\prime}}\right\rangle=\frac{\int d \Gamma q_{k}^{*} q_{k^{\prime}}\left(\lambda^{2} V^{2} / 2\right) \exp \left(-\beta H_{0}\right)}{\int d \Gamma \exp \left(-\beta H_{0}\right)}+O\left(\lambda^{4}\right) \\
=\lambda^{2} V_{k} V_{k^{\prime}} \frac{1}{\beta \omega_{1}} \frac{1}{\beta \omega_{k}} \frac{1}{\beta \omega_{k^{\prime}}}+O\left(\lambda^{4}\right)
\end{gathered}
$$

Noting that $V_{k} \sim L^{-1 / 2}$, and $\sum_{k^{\prime}} \sim O(L)$, we obtain the result (81). One can check as well that terms of higher order in $\lambda V$ retain the same volume dependences.

For an ensemble of the form Eq. (41), we have $\left\langle q_{k}^{*} q_{k^{\prime}}\right\rangle=$ 0 , while $\left\langle J_{k}\right\rangle \sim O(1)$. This is a special case of Eq. (81).

\section{APPENDIX F: $\Lambda$ AND PRESERVATION OF THE MEASURE}

From the requirement (2) in the Introduction, $\Lambda$ preserves the measure of phase space. This means that

$$
\begin{aligned}
& \int d \Gamma \Lambda \rho=1 \\
& \int d \Gamma \Lambda^{\dagger} \rho=1
\end{aligned}
$$

for any normalized ensemble $\rho$. The first equality is easily shown, since it may be written as

$$
\int d \Gamma\left(\Lambda^{\dagger} 1\right) \rho=1
$$


where 1 is the unit operator. Since $\Lambda^{\dagger}$ can be expressed as a perturbation expansion,

$$
\Lambda^{\dagger}=1+O\left(\lambda L_{V}\right)
$$

and $L_{V} 1=0$ we conclude that $\Lambda^{\dagger} 1=1$, from which Eq. (F1) follows. Similarly, one can show the second equality (F2).

So, Eq. (F2) should be satisfied for the specific $\Lambda$ we have constructed in Sec. VI This condition will allow us to derive the relation (88). Consider the ensemble

$$
\rho=C_{1} q_{1}^{*} q_{1} \exp \left(-J / J_{0}\right)
$$

where $C_{1}$ is the normalization factor given by

$$
C_{s}=\left[\int d \Gamma q_{s}^{*} q_{s} \exp \left(-J / J_{0}\right)\right]^{-1}
$$

with

$$
J=\sum_{s} q_{s}^{*} q_{s}
$$

and $J_{0}$ a constant that makes the argument of the exponential dimensionless. The factor $\exp \left(-J / J_{0}\right)$ ensures the existence of a finite norm of $\rho$, (see the SegalBargmann representation in [22, 46]). The total action $J$ is an invariant of motion, because we have $L_{0} J=0$ and $L_{V} J=0$. Using Eq. (F4), we get

$$
\Lambda^{\dagger} J=J, \quad \Lambda^{\dagger} \exp \left(-J / J_{0}\right)=\exp \left(-J / J_{0}\right) .
$$

The operator $L_{V}$ is a differential operator. Applying the chain rule of differentiation and Eq. (F8) we conclude that

$$
\Lambda^{\dagger} q_{1}^{*} q_{1} \exp \left(-J / J_{0}\right)=\left(\Lambda^{\dagger} q_{1}^{*} q_{1}\right) \exp \left(-J / J_{0}\right) .
$$

Inserting the ensemble Eq. (F5) in Eq. (F2) and using Eq. (86) we get

$$
\begin{aligned}
& C_{1} \int d \Gamma\left|N_{1}\right|\left[q_{1}^{*} q_{1}+\lambda^{2} \sum_{k}\left(r c_{k}^{2}+\text { c.c. }\right) q_{k}^{*} q_{k}\right] \\
& \times \exp \left(-J / J_{0}\right)=1 .
\end{aligned}
$$

where the off-diagonal terms such as $q_{1}^{*} q_{k}$ appearing in the product $\tilde{Q}_{1}^{*} \tilde{Q}_{1}$ in Eq. (86) vanish due to the integration over angles in phase space. We can write Eq. (F10) as

$$
C_{1}\left|N_{1}\right|\left[C_{1}^{-1}+\lambda^{2} \sum_{k}\left(r c_{k}^{2}+\text { c.c. }\right) C_{k}^{-1}\right]=1 .
$$

Since $C_{1}=C_{k}$ for any $k$ (see Eq. (F6) $)$, Eq. (F10) leads to

$$
\left|N_{1}\right|\left[1+\lambda^{2} \sum_{k}\left(r c_{k}^{2}+\text { c.c. }\right)\right]=1 .
$$

This equation plus the condition $r+r^{*}=1$ yield the result (88). With this result we can write

$$
\Lambda^{\dagger} q_{1}^{*} q_{1}=Q^{(0)} \tilde{Q}_{1}^{*} \tilde{Q}_{1}+P^{(0)}\left(r \tilde{Q}_{1}^{*} Q_{1}+\text { c.c. }\right)
$$

where $P^{(0)}$ is the projector to angle-independent monomials (such as $q_{s}^{*} q_{s}$ ) and $Q^{(0)}=1-P^{(0)}$. This shows that the transformed product of particle modes can be simply written as a combination of renormalized particle modes (i.e., Gamow modes).

The derivation followed here is similar to the derivation followed in Ref. 15], where we used the $\Lambda$ transformation to define dressed unstable states in quantum mechanics. The only difference is that in [15] the relation $r+r^{*}=1$ was derived from the requirement that the dressed unstable state has an energy fluctuation of the order of the inverse lifetime. This fluctuation is a quantum effect. Here we are dealing with classical mechanics, so we postulate $r+r^{*}=1$ as a basic condition. An alternative derivation, presented in Appendix A of Ref. 16], started with the analogue of Eq. (F13), as a postulate. All the derivations give the same result (88). Note that a condition different from $r+r^{*}=1$ would not allow us to express $\Lambda^{\dagger} q_{1}^{*} q_{1}$ as a combination of Gamow modes only, and it would lead to energy fluctuations different from the inverse lifetime in the quantum case, which would be unphysical.

The preservation of the measure can be proven for more general ensembles involving monomials of particle modes, which we have considered in Sec. VI Indeed, from the relation $\sum_{k} c_{k}^{*} c_{k}=-1$ (see [15]) we have (see Eqs. (F12, (87))

$$
\sum_{k} b_{k}=1
$$

Using this relation as well as the expression (91) one can show that for $\rho=C_{m n} q_{1}^{* m} q_{1}^{n} \exp \left(-J / J_{0}\right)$ with $C_{m n}$ a normalization constant, we have

$$
\int d \Gamma \Lambda^{\dagger} \rho=\int d \Gamma \rho=\delta_{m n} .
$$

The proof (which we will omit here) uses the relation $\int d \Gamma \tilde{Q}_{1}^{* m} \tilde{Q}_{1}^{n} \exp \left(-J / J_{0}\right)=0$. This follows from the fact that both $\tilde{Q}_{1}^{* m} \tilde{Q}_{1}^{n}$ and $\exp \left(-J / J_{0}\right)$ are eigenfunctions of $L_{H}$ with different eigenvalues, which implies their orthogonality.

\section{APPENDIX G: PROOF OF ANALYTICITY OF $\Lambda$}

In this appendix we show that Eq. (91) removes all the non-analytic $\left|c_{k}\right|^{2}$ terms, replacing them by $\xi_{k}=r c_{k}^{2}+$ c.c. First we derive recursive formulas to calculate $\Lambda^{\dagger} q_{1}^{* m} q_{1}^{n}$. We start with Eq. (91) for $m \geq n$

$$
\Lambda^{\dagger} q_{1}^{* m} q_{1}^{n}=\sum_{l=0}^{n} \frac{m ! n !}{(m-l) !(n-l) ! l !} \tilde{Q}_{1}^{* m-l} \tilde{Q}_{1}^{n-l} Y^{l} .
$$

(the $n>m$ case can be calculated by taking the complex conjugate of Eq. (G1)). We have as well

$$
\Lambda^{\dagger} q_{1}^{* m+1} q_{1}^{n}
$$




$$
=\sum_{l=0}^{n} \frac{(m+1) ! n !}{(m+1-l) !(n-l) ! l !} \tilde{Q}_{1}^{* m+1-l} \tilde{Q}_{1}^{n-l} Y^{l}
$$

For $l>0$ we have the identity

$$
\frac{(m+1) !}{(m+1-l) ! l !}=\frac{m !}{(m-l) ! l !}+\frac{m !}{(m+1-l) !(l-1) !}
$$

Inserting this in Eq. G2 we get

$$
\begin{aligned}
& \Lambda^{\dagger} q_{1}^{* m+1} q_{1}^{n}=\tilde{Q}_{1}^{* m+1} \tilde{Q}_{1}^{n} \\
& +\sum_{l=1}^{n}\left[\frac{m !}{(m-l) ! l !}+\frac{m !}{(m+1-l) !(l-1) !}\right] \\
& \times \frac{n !}{(n-l) !} \tilde{Q}_{1}^{* m+1-l} \tilde{Q}_{1}^{n-l} Y^{l} .
\end{aligned}
$$

The first term plus the second term give

$$
\left(\Lambda^{\dagger} q_{1}^{* m} q_{1}^{n}\right) \Lambda^{\dagger} q_{1}^{*}
$$

(note that $\Lambda^{\dagger} q_{1}^{*}=\tilde{Q}_{1}^{*}$ ). The third term may be written as (with $l^{\prime}=l-1$ )

$$
\begin{aligned}
& \sum_{l^{\prime}=0}^{n-1} \frac{m !}{\left(m-l^{\prime}\right) !\left(l^{\prime}\right) !} \frac{n(n-1) !}{\left(n-l^{\prime}-1\right) !} \tilde{Q}_{1}^{* m-l^{\prime}} \tilde{Q}_{1}^{n-l^{\prime}-1} Y^{l^{\prime}+1} \\
& =n Y \Lambda^{\dagger} q_{1}^{* m} q_{1}^{n-1}
\end{aligned}
$$

Therefore

$$
\begin{aligned}
& \Lambda^{\dagger} q_{1}^{* m+1} q_{1}^{n} \\
& =\left(\Lambda^{\dagger} q_{1}^{* m} q_{1}^{n}\right) \Lambda^{\dagger} q_{1}^{*}+n Y \Lambda^{\dagger} q_{1}^{* m} q_{1}^{n-1} \quad(m \geq n) .
\end{aligned}
$$

For $m>n$ we have, from Eq. (G1),

$$
\begin{aligned}
\Lambda^{\dagger} q_{1}^{* m} q_{1}^{n+1} & =\sum_{l=0}^{n} \frac{m !(n+1) !}{(m-l) !(n+1-l) ! l !} \\
& \times \tilde{Q}_{1}^{* m-l} \tilde{Q}_{1}^{n+1-l} Y^{l} .
\end{aligned}
$$

Using Eq. G3 we get

$$
\begin{aligned}
& \Lambda^{\dagger} q_{1}^{* m} q_{1}^{n+1} \\
& =\tilde{Q}_{1}^{* m} \tilde{Q}_{1}^{n+1}+\frac{m !}{(m-n-1) !} \tilde{Q}_{1}^{* m-n-1} Y^{n+1} \\
& +\sum_{l=1}^{n} \frac{m !}{(m-l) !}\left[\frac{n !}{(n-l) ! l !}+\frac{n !}{(n+1-l) !(l-1) !}\right] \\
& \times \tilde{Q}_{1}^{* m-l} \tilde{Q}_{1}^{n+1-l} Y^{l} .
\end{aligned}
$$

Adding the first and the third terms we get

$$
\left(\Lambda^{\dagger} q_{1}^{* m} q_{1}^{n}\right) \Lambda^{\dagger} q_{1}
$$

(note that $\Lambda^{\dagger} q_{1}=\tilde{Q}_{1}$ ). Adding the second and fourth terms we get (with $l^{\prime}=l-1$ )

$$
\begin{aligned}
& \sum_{l^{\prime}=0}^{n} \frac{m(m-1) !}{\left(m-l^{\prime}-1\right) !} \frac{n !}{\left(n-l^{\prime}\right) !\left(l^{\prime}\right) !} \tilde{Q}_{1}^{* m-l^{\prime}-1} \tilde{Q}_{1}^{n-l^{\prime}} Y^{l^{\prime}+1} \\
& =m Y \Lambda^{\dagger} q_{1}^{* m-1} q_{1}^{n} .
\end{aligned}
$$

Therefore

$$
\begin{aligned}
& \Lambda^{\dagger} q_{1}^{* m} q_{1}^{n+1} \\
& =\left(\Lambda^{\dagger} q_{1}^{* m} q_{1}^{n}\right) \Lambda^{\dagger} q_{1}+m Y \Lambda^{\dagger} q_{1}^{* m-1} q_{1}^{n} \quad(m>n) .
\end{aligned}
$$

Eqs. (G7) and (G12) plus their complex conjugates permit one to construct $\Lambda^{\dagger} q_{1}^{* m} q_{1}^{n}$ recursively.

Now we prove the analyticity of $\Lambda^{\dagger} q_{1}^{* m} q_{1}^{n}$ at $\lambda=0$ from the recursive relations. In the recursive relation, we show that if the lower order terms in $m$ and $n$ like $\Lambda^{\dagger} q^{* m} q_{1}^{n}, \Lambda^{\dagger} q_{1}^{* m} q_{1}^{n-1}$ and $\Lambda^{\dagger} q_{1}^{* m-1} q_{1}^{n-1}$ are analytic, then the higher order terms $\Lambda^{\dagger} q^{* m+1} q_{1}^{n}$ and $\Lambda^{\dagger} q_{1}^{* m} q_{1}^{n}$ are also analytic. Then from mathematical induction, the analyticity of $\Lambda^{\dagger} q_{1}^{* m} q_{1}^{n}$ is proved for general $m$ and $n$ (the $m<n$ case can be shown in the same way). In Eq. (G7),

$$
\begin{aligned}
& \Lambda^{\dagger} q_{1}^{* m+1} q_{1}^{n}=\left(\Lambda^{\dagger} q^{* m} q_{1}^{n}\right) \Lambda^{\dagger} q_{1}^{*}+n Y \Lambda^{\dagger} q_{1}^{* m} q_{1}^{n-1} \\
& =\left(\sum_{l=0}^{n} \frac{m ! n !}{(m-l) !(n-l) ! l !} \tilde{Q}_{1}^{* m-l} \tilde{Q}_{1}^{n-l} Y^{l}\right) \tilde{Q}_{1}^{*} \\
& +n Y\left(\sum_{l=0}^{n-1} \frac{m !(n-1) !}{(m-l) !(n-1-l) ! l !} \tilde{Q}_{1}^{* m-l} \tilde{Q}_{1}^{n-1-l} Y^{l}\right)
\end{aligned}
$$

Suppose that the quantities inside large parenthesis are analytic in $\lambda$. The additional non-analytic terms appear whenever additional products $\tilde{Q}_{1}^{*} \tilde{Q}_{1}$ appear. Since

$$
\tilde{Q}_{1}=N_{1}^{1 / 2}\left(q_{1}+\lambda \sum_{k} c_{k} q_{k}\right)
$$

each $\tilde{Q}_{1}^{*} \tilde{Q}_{1}$ produces a $\left|c_{k}\right|^{2}$ term, which is non-analytic in $\lambda$. Let us denote the non-analytic part of a function $f(\lambda)$ as $F n(f(\lambda))$. The non-analytic part in the first term in the right hand side of Eq. G13 is made by the additional $\tilde{Q}_{1}^{*}$ multiplied by $\tilde{Q}_{1}^{n-l}$, which generates $n-l$ terms $\left|c_{k}\right|^{2}$ :

$$
\begin{aligned}
& F n\left[\left(\sum_{l=0}^{n} \frac{m ! n !}{(m-l) !(n-l) ! l !} \tilde{Q}_{1}^{* m-l} \tilde{Q}_{1}^{n-l} Y^{l}\right) \tilde{Q}_{1}^{*}\right] \\
& =\sum_{l=0}^{n} \frac{m ! n !}{(m-l) !(n-l) ! l !} \tilde{Q}_{1}^{* m-l} \tilde{Q}_{1}^{n-l-1} Y^{l} \\
& \times(n-l) \lambda^{2}\left|N_{1}\right| \sum_{k}\left|c_{k}\right|^{2} q_{k}^{*} q_{k} \\
& =\sum_{l=0}^{n-1} \frac{m ! n !}{(m-l) !(n-l-1) ! l !} \tilde{Q}_{1}^{* m-l} \tilde{Q}_{1}^{n-l-1} Y^{l} \\
& \times \lambda^{2}\left|N_{1}\right| \sum_{k}\left|c_{k}\right|^{2} q_{k}^{*} q_{k} .
\end{aligned}
$$

The non-analytic part in the second term in the right hand side of Eq. G13 is coming from $Y$. Since

$$
\begin{aligned}
& Y=\sum_{k} b_{k} q_{k}^{*} q_{k} \\
& =\sum_{k} \lambda^{2}\left|N_{1}\right|\left(-\left|c_{k}\right|^{2}+r c_{k}^{2}+r^{*} c_{k}^{* 2}\right) q_{k}^{*} q_{k},
\end{aligned}
$$


the non-analytic function $\left|c_{k}\right|^{2}$ appears inside $Y$.

$$
\begin{aligned}
& F n\left[n Y \sum_{l=0}^{n-1} \frac{m !(n-1) !}{(m-l) !(n-1-l) ! l !} \tilde{Q}_{1}^{* m-l} \tilde{Q}_{1}^{n-1-l} Y^{l}\right] \\
& =-\lambda^{2}\left|N_{1}\right| \sum_{k}\left|c_{k}\right|^{2} q_{k}^{*} q_{k} \\
& \times \sum_{l=0}^{n-1} \frac{m ! n !}{(m-l) !(n-1-l) ! a !} \tilde{Q}_{1}^{* m-l} \tilde{Q}_{1}^{* n-1-l} Y^{l} \cdot(\mathrm{G} 17)
\end{aligned}
$$

The non-analytic parts from the first term and second term in Eq. G13 exactly cancels out. So, the left hand side of Eq. G13 is analytic in $\lambda$. Note that terms of the form $\left|c_{k}\right|^{2 n}$ with $n>1$ give $O(1 / L)$ contributions and thus they are negligible.

Next, we show that the left hand side of Eq. G12 is analytic in $\lambda$. The non-analytic part of the first term in the right hand side of Eq. G12 is

$$
\begin{aligned}
& F n\left[\left(\Lambda^{\dagger} q_{1}^{* m} q_{1}^{n}\right) \Lambda^{\dagger} q_{1}\right] \\
& =F n\left[\left(\sum_{l=0}^{n} \frac{m ! n !}{(m-l) !(n-l) ! l !} \tilde{Q}_{1}^{* m-l} \tilde{Q}_{1}^{n-l} Y^{l}\right) \tilde{Q}_{1}\right] \\
& =\sum_{l=0}^{n} \frac{m ! n !}{(m-l) !(n-l) ! l !} \tilde{Q}_{1}^{* m-l-1} \tilde{Q}_{1}^{n-l} Y^{l} \\
& \times(m-l) \lambda^{2}\left|N_{1}\right| \sum_{k}\left|c_{k}\right|^{2} q_{k}^{*} q_{k} \\
& =\sum_{l=0}^{n} \frac{m ! n !}{(m-l-1) !(n-l) ! l !} \tilde{Q}_{1}^{* m-l-1} \tilde{Q}_{1}^{n-l} Y^{l} \\
& \times \lambda^{2}\left|N_{1}\right| \sum_{k}\left|c_{k}\right|^{2} q_{k}^{*} q_{k}
\end{aligned}
$$

The non-analytic part of the second term in the right hand side of Eq. G12 is

$$
\begin{aligned}
& F n\left[m Y \Lambda^{\dagger} q_{1}^{* m-1} q_{1}^{n}\right] \\
& =-m \lambda^{2}\left|N_{1}\right| \sum_{k}\left|c_{k}\right|^{2} q_{k}^{*} q_{k} \\
& \times \sum_{l=0}^{n} \frac{(m-1) ! n !}{(m-l-1) !(n-l) ! l !} \tilde{Q}_{1}^{* m-1-l} \tilde{Q}_{1}^{n-l} Y^{l} \\
& =-\lambda^{2}\left|N_{1}\right| \sum_{k}\left|c_{k}\right|^{2} q_{k}^{*} q_{k} \\
& \times \sum_{l=0}^{n} \frac{m ! n !}{(m-l-1) !(n-l) ! l !} \tilde{Q}_{1}^{* m-1-l} \tilde{Q}_{1}^{n-l} Y^{l} .
\end{aligned}
$$

Again, the non-analytic parts of the first and second terms of Eq. G12 exactly cancel out. The right hand side of Eq. G12 is analytic in $\lambda$. Therefore from the mathematical induction $\Lambda^{\dagger} q_{1}^{* m} q_{1}^{n}$ is analytic in $\lambda$.

\section{APPENDIX H: CALCULATION OF THE NOISE CONSTANT $\hat{R}_{c}$}

In this appendix we determine the noise constant $\hat{R}_{c}$. We assume that the noise $\hat{R}(t)$ comes from the thermal bath with temperature $T$. In this case, we expect that the system reaches thermal equilibrium for $t \rightarrow \infty$. Furthermore, from the equipartition theorem we expect that

$$
\frac{1}{2} \hat{m} \hat{\omega}_{1}^{2}\left\langle\hat{x}_{1}^{2}\right\rangle_{e q}=\frac{\left\langle\hat{p}_{1}^{2}\right\rangle_{e q}}{2 \hat{m}}=\frac{1}{2} k_{B} T,
$$

where $k_{B}$ is Boltzmann's constant. Substituting the relations

$$
\begin{aligned}
& \hat{x}_{1}(t)=\sqrt{\frac{1}{2 m_{p} \hat{\omega}_{1}}}\left(\hat{q}_{1}(t)+\hat{q}_{1}^{*}(t)\right), \\
& \hat{p}_{1}(t)=-i \sqrt{\frac{m_{p} \hat{\omega}_{1}}{2}}\left(\hat{q}_{1}(t)-\hat{q}_{1}^{*}(t)\right)
\end{aligned}
$$

into Eq. (H1), we get the conditions

$$
\begin{aligned}
& \left\langle\hat{q}_{1}^{2}(t)\right\rangle_{e q}+\left\langle\hat{q}_{1}^{* 2}(t)\right\rangle_{e q}=0, \\
& \hat{\omega}_{1}\left\langle\hat{q}_{1}^{*}(t) \hat{q}_{1}(t)\right\rangle_{e q}=k_{B} T .
\end{aligned}
$$

On the other hand we have

$$
\begin{aligned}
& \left\langle\hat{q}_{1}^{*}(t) \hat{q}_{1}(t)\right\rangle_{e q} \\
& =\lim _{t \rightarrow \infty}\left(\left\langle\hat{q}_{1 a}^{*}(t) \hat{q}_{1 a}(t)\right\rangle+\left\langle\hat{q}_{1 r}^{*}(t) \hat{q}_{1 r}(t)\right\rangle\right) \\
& =\lim _{t \rightarrow \infty}\left\langle\hat{q}_{1 r}^{*}(t) \hat{q}_{1 r}(t)\right\rangle, \\
& \left\langle\hat{q}_{1 a}^{*}(t) \hat{q}_{1 r}(t)\right\rangle \\
& =\left\langle e^{-2 \hat{\gamma} t} \int_{0}^{t} \int_{0}^{t} d t_{1} d t_{2} \hat{R}\left(t_{1}\right) \hat{R}^{*}\left(t_{2}\right)\right. \\
& \left.\times e^{\left.i \hat{z}_{1} t_{1}-1 \hat{z}_{1}^{*} t_{2}\right)}\right\rangle \\
& =e^{-2 \hat{\gamma} t} \int_{0}^{t} d t_{1} \hat{R}_{c}^{2} e^{2 \hat{\gamma} t_{1}} \frac{\hat{R}_{c}^{2}\left(1-e^{-2 \hat{\gamma} t}\right)}{2 \hat{\gamma}} .
\end{aligned}
$$

Substituting this to Eq. (H4), we get

$$
\hat{R}_{c}^{2}=\frac{2 \hat{\gamma} k_{B} T}{\hat{\omega}_{1}}
$$

\section{APPENDIX I: CALCULATION OF THE MOMENTS}

In this appendix we calculate the moments in Eq. (124). We have

$$
\begin{aligned}
& \int d \Gamma\left(q_{1}^{*}-q_{1}^{\prime}{ }^{*}\right)^{m}\left(q_{1}-q_{1}^{\prime}\right)^{n} \tilde{\theta}(\Gamma) \delta\left(\Gamma-\Gamma^{\prime}\right) \\
& =\int d \Gamma\left[\tilde{\theta}^{\dagger}(\Gamma)\left(q_{1}^{*}-q_{1}^{*}\right)^{n}\left(q_{1}-q_{1}^{\prime}\right)^{m}\right]^{*} \delta\left(\Gamma-\Gamma^{\prime}\right) \\
& =-\int d \Gamma\left[\left(\Lambda^{\dagger}\right)^{-1} L_{H} \Lambda^{\dagger}\left(q_{1}^{*}-q_{1}^{\prime}\right)^{m}\left(q_{1}-q_{1}^{\prime}\right)^{n}\right] \delta\left(\Gamma-\Gamma^{\prime}\right),
\end{aligned}
$$


where we used the relation $L_{H}^{\dagger}=L_{H}$ and $L_{H}^{*}=-L_{H}$. The quantity inside the brackets in Eq. (II) [which we call $I]$ is

$$
\begin{aligned}
& I=\left(\Lambda^{\dagger}\right)^{-1} L_{H} \Lambda^{\dagger}\left(q_{1}^{*}-q_{1}^{\prime *}\right)^{m}\left(q_{1}-q_{1}^{\prime}\right)^{n} \\
& =-\left.i \frac{d}{d t}\left(\Lambda^{\dagger}\right)^{-1} e^{i L_{H} t} \Lambda^{\dagger}\left(q_{1}^{*}-q_{1}^{\prime *}\right)^{m}\left(q_{1}-q_{1}^{\prime}\right)^{n}\right|_{t=0} \\
& =\sum_{l=0}^{m} \sum_{j=0}^{n}\left(-q_{1}^{\prime *}\right)^{l}\left(-q_{1}^{\prime}\right)^{j} \frac{m ! n !}{(m-l) !(n-j) ! l ! j !} \\
& \times\left.\left(-i \frac{d}{d t}\right)\left(\Lambda^{\dagger}\right)^{-1} e^{i L_{H} t} \Lambda^{\dagger} q_{1}^{* m-l} q_{1}^{n-j}\right|_{t=0}
\end{aligned}
$$

Using Eq. (115), we have

$$
\begin{aligned}
& I=\sum_{l=0}^{m} \sum_{j=0}^{n}\left(-q_{1}{ }^{*}\right)^{l}\left(-q_{1}^{\prime}\right)^{j} \frac{m ! n !}{(m-l) !(n-j) ! l ! j !}\left(-i \frac{d}{d t}\right) \\
& \times \sum_{a=0}^{m i n(m-l, n-l)} \frac{(m-l) !(n-l) !}{(m-l-a) !(n-j-a) ! a !} \\
& \times e^{i\left((m-l) z_{1}^{*}-(n-j) z_{1}\right) t} q_{1}^{* m-l-a} q_{1}^{n-j-a} Y^{a} \\
& \times\left.\left(e^{2 \gamma t}-1\right)^{a}\right|_{t=0} .
\end{aligned}
$$

Because of the $\left(e^{2 \gamma t}-1\right)^{a}$ term, the only non-vanishing terms in Eq. (13) at $t=0$ are for $a=0$ or $a=1$. So the above equation becomes

$$
\begin{aligned}
& I=\sum_{l=0}^{m} \sum_{j=0}^{n}\left(-q_{1}^{\prime}\right)^{l}\left(-q_{1}^{\prime}\right)^{j} \frac{m ! n !}{l ! j !(m-l) !(n-j) !} \\
& \times\left.\left(-i \frac{d}{d t}\right)\left(e^{i z_{1}^{*} t} q_{1}^{*}\right)^{m-l}\left(e^{-i z_{1} t} q_{1}\right)^{n-j}\right|_{t=0} \\
& +\sum_{l=0}^{m-1} \sum_{j=0}^{n-1}\left(-q_{1}^{\prime *}\right)^{l}\left(-q_{1}^{\prime}\right)^{j} \frac{m ! n !}{l ! j !(m-l-1) !(n-j-1) !} \\
& \times\left(-i \frac{d}{d t}\right) e^{i\left(z_{1}-z_{1}^{*}\right) t}\left(e^{i z_{1}^{*} t} q_{1}^{*}\right)^{m-l-1}\left(e^{-i z_{1} t} q_{1}\right)^{n-j-1} Y \\
& \times\left.\left(e^{2 \gamma t}-1\right)\right|_{t=0} \\
& =\left.\left(-i \frac{d}{d t}\right)\left(e^{i z_{1}^{*} t} q_{1}^{*}-q_{1}^{*}\right)^{m}\left(e^{-i z_{1} t} q_{1}-q_{1}^{\prime}\right)^{n}\right|_{t=0} \\
& +\left(-i \frac{d}{d t}\right) m n Y\left(1-e^{-2 \gamma t}\right) \\
& \times\left.\left(e^{i z_{1}^{*} t} q_{1}^{*}-q_{1}^{\prime *}\right)^{m-1}\left(e^{-i z_{1} t} q_{1}-q_{1}^{\prime}\right)^{n-1}\right|_{t=0} .
\end{aligned}
$$

Substituting Eq. (I4) into Eq. (I1) and integrating with $\delta\left(\Gamma-\Gamma^{\prime}\right)$, we get Eq. (124).

\section{APPENDIX J: FACTORIZATION PROPERTY}

We show the factorization of Eq. (128) when $\tilde{\rho}(\Gamma, 0)$ has the form

$$
\tilde{\rho}(\Gamma, 0)=g_{1}\left(q_{1}^{*}, q_{1}\right) \prod_{k} g_{k}\left(q_{k}^{*}, q_{k}\right) .
$$

In Eq. (128), by integrating by parts, we can write

$$
\begin{aligned}
& \int d \Gamma G\left(q_{1}, q_{1}^{*}\right) \frac{\partial^{2}}{\partial q_{1} \partial q_{1}^{*}} \sum_{k} b_{k} J_{k} \tilde{\rho}(\Gamma, t) \\
& =\int d \Gamma \frac{\partial^{2}}{\partial q_{1} \partial q_{1}^{*}} G\left(q_{1}, q_{1}^{*}\right) \sum_{k} b_{k} J_{k} \tilde{\rho}(\Gamma, t) \\
& =\int d \Gamma\left(\frac{\partial^{2}}{\partial q_{1} \partial q_{1}^{*}} G\left(q_{1}, q_{1}^{*}\right)\right) \sum_{k} b_{k} J_{k} e^{-i \tilde{\theta} t} \tilde{\rho}(\Gamma, 0) .
\end{aligned}
$$

Let us expand

$$
\frac{\partial^{2}}{\partial q_{1} \partial q_{1}^{*}} G\left(q_{1}, q_{1}^{*}\right)=\sum_{m, n} G_{m n} q_{1}^{* m} q_{1}^{n} .
$$

We have

$$
\begin{aligned}
& \int d \Gamma q_{1}^{* m} q_{1}^{n} \sum_{k} b_{k} J_{k} e^{-i \tilde{\theta} t} \tilde{\rho}(\Gamma, 0) \\
& =\int d \Gamma\left[\left(e^{-i \tilde{\theta} t}\right)^{\dagger} q_{1}^{m} q_{1}^{* n} \sum_{k} b_{k} J_{k}\right]^{*} \tilde{\rho}(\Gamma, 0) .
\end{aligned}
$$

Since

$$
L_{0} \sum_{k} b_{k} J_{k}=0, \quad L_{V} \sum_{k} b_{k} J_{k}=O(1 / \sqrt{L})
$$

and $\Lambda$ is expressed in terms of $L_{0}$ and $L_{V}, \tilde{\theta}=\Lambda L_{H} \Lambda^{-1}$ treats $\sum_{k} b_{k} J_{k}$ like constant. Neglecting $O(1 / \sqrt{L})$ terms, we can write

$$
\begin{aligned}
& \int d \Gamma q_{1}^{* m} q_{1}^{n} \sum_{k} b_{k} J_{k} e^{-i \tilde{\theta} t} \tilde{\rho}(\Gamma, 0) \\
& =\int d \Gamma \sum_{k} b_{k} J_{k}\left[\left(e^{-i \tilde{\theta} t}\right)^{\dagger} q_{1}^{m} q_{1}^{* n}\right]^{*} \tilde{\rho}(\Gamma, 0) .
\end{aligned}
$$

In Eq. (J6), $\left[\left(e^{-i \tilde{\theta} t}\right)^{\dagger} q_{1}^{m} q_{1}^{* n}\right]^{*}$ can be written as (see Eq. (115)

$$
\begin{aligned}
& {\left[\left(e^{-i \tilde{\theta} t}\right)^{\dagger} q_{1}^{m} q_{1}^{* n}\right]^{*}=\left(\Lambda^{\dagger}\right)^{-1}\left(e^{i L_{H} t} \Lambda^{\dagger} q_{1}^{* m} q_{1}^{n}\right)} \\
& =\sum_{l=0}^{m i n(m, n)} \frac{m ! n !}{(m-l) !(n-l) ! l !} \\
& \times e^{i\left(m z_{1}^{*}-n z_{1}\right) t} q_{1}^{* m-l} q_{1}^{n-l} Y^{l}\left(e^{2 \gamma t}-1\right)^{l} .
\end{aligned}
$$

Since

$$
\sum_{k} b_{k} J_{k}\left(\sum_{k} b_{k} J_{k}\right)^{l}=\sum_{k} b_{k} J_{k}\left(\sum_{k^{\prime} \neq k} b_{k^{\prime}} J_{k^{\prime}}\right)^{l}+O(1 / L),
$$

we can write

$$
\begin{aligned}
& \sum_{k} b_{k} J_{k}\left[\left(e^{-i \tilde{\theta} t}\right)^{\dagger} q_{1}^{m} q_{1}^{* n}\right]^{*} \\
& =\sum_{k} b_{k} J_{k}\left[\left(e^{-i \tilde{\theta} t}\right)^{\dagger} q_{1}^{m} q_{1}^{* n}\right]_{f-k}^{*}+O(1 / L) .
\end{aligned}
$$


In Eq. (J9), [ $]_{f-k}$ means that we exclude the $k$ th field mode. With Eq. (J9) and neglecting $O(1 / L)$ terms, Eq. (J6) becomes

$$
\begin{aligned}
& \int d \Gamma \sum_{k} b_{k} J_{k}\left[\left(e^{-i \tilde{\theta} t}\right)^{\dagger} q_{1}^{m} q_{1}^{* n}\right]^{*} \tilde{\rho}(\Gamma, 0) \\
& =\sum_{k} \int d \Gamma b_{k} J_{k}\left[\left(e^{-i \tilde{\theta} t}\right)^{\dagger} q_{1}^{m} q_{1}^{* n}\right]_{f-k}^{*} \tilde{\rho}(\Gamma, 0) \\
& =\sum_{k} \int d \Gamma b_{k} J_{k}\left[\left(e^{-i \tilde{\theta} t}\right)^{\dagger} q_{1}^{m} q_{1}^{* n}\right]_{f-k}^{*} g_{1}\left(\Gamma_{1}\right) \prod_{k} g_{k}\left(\Gamma_{k}\right) \\
& =\sum_{k} \int d \Gamma_{k} b_{k} J_{k} g_{k}\left(\Gamma_{k}\right) \\
& \times \int d \Gamma_{f-k}\left[\left(e^{-i \tilde{\theta} t}\right)^{\dagger} q_{1}^{m} q_{1}^{* n}\right]_{f-k}^{*} g_{1}\left(\Gamma_{1}\right) \prod_{k^{\prime} \neq k} g_{k^{\prime}}\left(\Gamma_{k^{\prime}}\right) .
\end{aligned}
$$

For any $k$ we have

$$
\int d \Gamma_{k} g\left(\Gamma_{k}\right)=1 .
$$

Then we can write

$$
\int d \Gamma_{k} b_{k} J_{k} g_{k}\left(\Gamma_{k}\right)
$$

$$
\begin{aligned}
& =\int d \Gamma b_{k} J_{k} g_{1}\left(\Gamma_{1}\right) \prod_{k} g_{k}\left(\Gamma_{k}\right)=b_{k}\left\langle J_{k}\right\rangle \\
& \int d \Gamma_{f-k}\left[\left(e^{-i \tilde{\theta} t}\right)^{\dagger} q_{1}^{m} q_{1}^{* n}\right]_{f-k}^{*} g_{1}\left(\Gamma_{1}\right) \prod_{k^{\prime} \neq k} g_{k^{\prime}}\left(\Gamma_{k^{\prime}}\right) \\
& =\int d \Gamma\left[\left(e^{-i \tilde{\theta} t}\right)^{\dagger} q_{1}^{m} q_{1}^{* n}\right]_{f-k}^{*} g_{1}\left(\Gamma_{1}\right) \prod_{k^{\prime}} g_{k^{\prime}}\left(\Gamma_{k^{\prime}}\right) \\
& =\int d \Gamma q_{1}^{* m} q_{1}^{n} e^{-i \tilde{\theta} t} \tilde{\rho}(\Gamma, 0),
\end{aligned}
$$

and Eq. J10 can be written as

$$
\begin{aligned}
& \int d \Gamma \sum_{k} b_{k} J_{k}\left[\left(e^{-i \tilde{\theta} t}\right)^{\dagger} q_{1}^{m} q_{1}^{* n}\right]^{*} \tilde{\rho}(\Gamma, 0) \\
& =\sum_{k} b_{k}\left\langle J_{k}\right\rangle \int d \Gamma q_{1}^{* m} q_{1}^{n} e^{-i \tilde{\theta} t} \tilde{\rho}(\Gamma, 0) \\
& =\sum_{k} b_{k}\left\langle J_{k}\right\rangle \int d \Gamma q_{1}^{* m} q_{1}^{n} \tilde{\rho}(\Gamma, t) .
\end{aligned}
$$

This equation, together with Eqs. (J2) and (J3), leads to Eq. (128).
[1] R. D. Mattuck, A guide to Feynman diagrams in the many-body problem, second edition (Dover, New York, 1992).

[2] K. Friedrichs, Commun. Pure Appl. Math. 1, 361 (1948).

[3] A. O. Caldeira and A. J. Leggett, Ann. Phys. 149, 374 (1983).

[4] C. W. Gardiner, Quantum Noise (Springer Verlag, 1991).

[5] E. Fick and G. Sauermann, The Quantum statistics of dynamical processes (Springer Verlag, 1990).

[6] U. Weiss, Quantum dissipative Systems, Chapters 2-3 (World Scientific, Singapore, 1983).

[7] Th. M. Nieuwenhuizen and A. E. Allahverdyan, arXiv: cond-mat/0011389 (2001).

[8] B. L. Hu, J. P. Paz and Y. Zhang, Phys. Rev. D, 45, 2843 (1992).

[9] E. Calzetta, A. Roura and E. Verdaguer, arXiv: quant-ph/0011097 (2001).

[10] I. Prigogine, From being to becoming (Freeman, New York, 1980).

[11] I. Prigogine, C. George, F. Henin, L. Rosenfeld, Chemica Scripta 4, 5 (1973).

[12] T. Petrosky and I. Prigogine, Physica A 175, 146 (1991).

[13] T. Petrosky and I. Prigogine, Chaos Solitons Fractals 7, 441 (1996).

[14] T. Petrosky and I. Prigogine, Adv. Chem. Phys. 99, 1 (1997).

[15] G. Ordonez, T. Petrosky and I. Prigogine, Phys. Rev. A 63, 052106 (2001).

[16] T. Petrosky, G. Ordonez and I. Prigogine, Phys. Rev. A 64, 062101 (2001).

[17] T. Petrosky and V. Barsegov, Phys. Rev. E 65, 046102 (2000).
[18] T. Petrosky and I. Prigogine, Chaos, Soliton and Fractals 11, 373 (2000).

[19] B. Misra and E.C.G. Sudarshan, J. Math. Phys. 18, 756 (1977).

[20] L. A. Khalfin, Sov. Phys. JETP 6, 1053 (1958).

[21] I. Prigogine and T. Petrosky, Physica A 147, 461 (1988).

[22] T. Petrosky, G. Ordonez, and I. Prigogine, to be published in Phys. Rev. A.

[23] C. Compagno, G. M. Palma, R. Passante and F. Persico, J. Phys. B 28, 1105 (1995).

[24] C. Cohen-Tannouji, J. Dupont-Roc and G. Grynberg, Atom-photon interactions. Basic processes and applications (Wiley, New York, 1992).

[25] W. T. Coffey, Yu. P. Kalmykov, J. T. Waldron, The Langevin equation with application in physics, chemistry and electrical engineering, (World Scientific, 1996).

[26] R. Balescu Equilibrium and nonequilibrium statistical mechanics, (John Wiley \& Sons, 1975).

[27] E.C.G. Sudarshan, C.B. Chiu and V. Gorini, Phys. Rev. D 18, 2914 (1978).

[28] N. Nakanishi, Prog. Theor. Phys. 19, 607 (1958).

[29] A. Böhm and M. Gadella, Dirac Kets, Gamow Vectors and Gelfand Triplets, (Springer Lecture Notes on Physics, Vol. 348, Springer, New York, 1989).

[30] T. Petrosky, I. Prigogine and S. Tasaki, Physica A 173, 175 (1991).

[31] S. Tasaki, Phys. Reports 219, 110 (1992).

[32] I. Antoniou, M. Gadella, E. Karpov, I. Prigogine, and G. Pronko, Chaos, Solitons and Fractals 12, 2757 (2001).

[33] T. Petrosky, private communication.

[34] T. Petrosky and I. Prigogine, Physica A 147, 439 (1988).

[35] I. Prigogine, Non Equilibrium Statistical Mechanics (Wi- 
ley Interscience, 1962).

[36] S. Kim, "Star unitary transformation and stochasticity: emergence of white, $1 / f$ noise through resonances," Dissertation, The University of Texas at Austin (2002).

[37] P. Resibois and M. de Leener, Classical kinetic theory of fluids (Wiley Interscience, 1977).

[38] N. G. van Kampen, Stochastic processes in physics and chemistry (North Holland Publishing Company, 1981).

[39] E. Nelson, Dynamical theories of Brownian motion, (Mathematical notes, Princeton University Press, 1967).

[40] L. Van Hove, Physica 23, 441 (1957).

[41] R. Brout and I. Prigogine, Physica 22, 621 (1956).

[42] L. Accardi and S. V. Kozyrev, Adv. Chem. Phys. 122, 215 (2002).

[43] J. L. Lebowitz and E. Rubin, Phys. Rev. 131, 2381 (1963).
[44] P. Resibois and R. Davis, Physica 30, 1077 (1964).

[45] J. L. Lebowitz and P. Resibois, Phys. Rev. 139, A 1101 (1965).

[46] J. R. Klauder and E. C. G. Sudarshan, Fundamentals of Quantum Optics, (V. A. Benjamin, Inc., New York, 1968).

[47] Neglecting $O(1 / L)$ terms, the second term in Eq. 866 may be expressed in terms of renormalized field modes as $\sum_{k} b_{k} \tilde{Q}_{k}^{*} \tilde{Q}_{k}$ (see Eq. A9)

[48] Note that variables $A$ evolve as $\exp \left(i L_{H} t\right) A$, while states $\rho$ evolve as $\exp \left(-i L_{H} t\right) \rho$. In Refs. 15, 16, 22] we considered transformed states that decay for $t>0$. For this reason in those papers we used the $\Lambda^{-1}$ transformation rather than $\Lambda^{\dagger}$ (see Eqs. (71), (76)). 